\title{
Buoyancy reversal in cloud-top mixing layers
}

\author{
Juan Pedro Mellado, ${ }^{\mathrm{a} *}$ Bjorn Stevens, ${ }^{\mathrm{b}, \mathrm{c}}$ Heiko Schmidt ${ }^{\mathrm{d}}$ and Norbert Peters ${ }^{\mathrm{a}}$ \\ ${ }^{a}$ Institut für Technische Verbrennung, RWTH Aachen University, Germany \\ ${ }^{\mathrm{b}}$ Dept. of Atmospheric Sciences, University of California, Los Angeles, USA \\ ${ }^{\mathrm{c}}$ Max Planck Institute for Meteorology, Hamburg, Germany \\ ${ }^{\mathrm{d}}$ Zuse Institute, Free University of Berlin, Germany
}

\begin{abstract}
A theoretical and numerical small-scale study of the evaporative cooling phenomenon that might appear in the stratocumulus-topped boundary layers is presented. An ideal configuration of a cloud-top mixing layer is considered as defined by two non-turbulent horizontal layers, stably stratified and with buoyancy reversal within a certain range of mixture fractions due to the evaporative cooling. Linear stability analysis of the shear-free configuration is employed to provide a new interpretation of the buoyancy reversal parameter, namely in terms of a time-scale ratio between the stable and the unstable modes of the system. An incompressible high-order numerical algorithm to perform direct numerical simulation of the configuration is described and two-dimensional simulations of single-mode perturbations are presented. These simulations confirm the role of the different parameters identified in the linear stability analysis and show that convoluted flow patterns can be generated by the evaporative cooling even for the low levels of buoyancy reversal found in stratocumulus clouds. They also show that there is no enhancement of turbulent entrainment of upper-layer fluid in the shear-free configuration, and turbulent mixing enhancement by the evaporative cooling is restricted to the lower layer. Copyright (C) 2009 Royal Meteorological Society
\end{abstract}

KEY WORDS buoyancy reversal instability; stratocumulus; free convection; free turbulent flows

Received 28 October 2008; Revised 5 February 2009; Accepted 6 March 2009

\section{Introduction}

The physical phenomena occuring at the stratocumulus top of the planetary boundary layer are well recognised as poorly understood (Stevens, 2002). This region normally separates a lower layer of turbulent flow from an upper layer of subsiding air that is approximately laminar, giving rise to turbulent entrainment, external intermittency and a turbulent/non-turbulent transition region, the so-called turbulence interface. This problem by itself, without any consideration of the cloud physics, is still a matter of basic research in the field of free turbulent flows, in spite of its importance and long-standing recognition (Corrsin and Kistler, 1955; Fernando, 1991; Dimotakis, 2005; Hunt et al., 2006). When the idiosyncrasies of the cloud, in this case stratocumulus, are added to it, the difficulty of the problem is almost overwhelming. However, accurate models of related quantities, like an entrainment rate or the subgrid-scale terms in large-eddy simulations, are necessary due to the role that these stratocumulus-top regions, of the order of metres or tens of metres (Caughey et al., 1982), play in larger-scale dynamics. In addition to the physical complexity of the problem, the range of scales it embodies confounds brute-force attempts to numerically explore the interplay between turbulent processes at the cloud top and those

\footnotetext{
${ }^{*}$ Correspondence to: J. P. Mellado, Institut für Technische Verbrennung, RWTH Aachen University, Templergrabenst. 64, 52056 Aachen, Germany. E-mail: jpmellado@itv.rwth-aachen.de
}

within the turbulent layer as a whole. Stevens (2002) reviews these issues and, as a part of the conclusion, advocates for the study of smaller-scale, simplified and more specific problems that would allow us to gain insight into the more general and more complex system. The authors adopt this approach in this paper and study in detail one aspect of the cloud-top mixing, namely, the role of latent heat effects in an infinite two-layer configuration.

Latent heat effects are expected to be important because of the evaporative cooling caused by the mixing between the lower cooler and saturated (and condensate-laden) layer and the upper warmer and unsaturated layer, which in some cases can lead to buoyancy reversal. The possibility of a resulting instability, the so-called cloud-top entrainment instability, which would ultimately break the cloud deck, remains an important and largely unresolved question (Randall, 1980; Deardorff, 1980; Kuo and Schubert, 1988; Grabowski, 1995; Wunsch, 2003; Yamaguchi and Randall, 2008).

Among the great number of studies focused on buoyancy reversal in cloud-topped layers, the series of papers by Shy and Breidenthal (1990), Siems et al. (1990) and Siems and Bretherton (1992) merit special attention. Shy and Breidenthal (1990) explore the effects of buoyancy reversal in a laboratory system (tank) consisting of methanol, ethylene glycol, salt and water, and characterized by two parameters: $D$, which measures the ratio of the maximum density change of the mixture to the density difference of the unmixed fluids; and $\chi_{\mathrm{s}}$, the 
mixture fraction at which that maximum density occurs. Siems et al. (1990) present two-dimensional numerical simulations of this two-layer configuration without explicit subgrid-scale modelling using a second-order algorithm. The evolution of the system is then described in terms of the stream function and the mixture fraction $\chi$ for the case of discrete initial perturbations in the form of a buoyancy anomaly. Although their low-order scheme is too dissipative and the Reynolds number affordable at that time possibly too small (which leads to an almost laminar structure of the reversing system for the case $D=0.05$ representative of real stratocumulus), their study was the first to recognize the applicability of direct numerical simulation (DNS) to this problem, as only DNS attempts to explicitly represent mixing processes and hence the scales at which latent heat effects are realized. These numerical studies were extended by Siems and Bretherton (1992) who tried to span the range between the laboratory scale, comparing with Shy and Breidenthal (1990) by using single-vortex initial conditions, and the atmospheric scale, using multiple-vortices initialization. A principal finding of these studies is the identification of a critical buoyancy reversal parameter $D$ of order unity beyond which a qualitative change of the flow (a runaway in the entrainment of dry fluid from the upper layer or a sustained increase of kinetic energy despite the viscous dissipation) becomes evident. Actual cloud-top conditions typically have $D \ll 1$, which leads them to conclude that buoyancy reversal alone is not likely to destabilize the cloud layer as a whole.

In this study we build on the results of this previous work by exploring two open questions. First, although the parameter $D$ can be easily introduced based on dimensional analysis, does it lend itself to a deeper physical interpretation? This is the topic of section 3, where a linear stability analysis is presented and used to interpret the buoyancy reversal parameter $D$ in terms of a ratio between the growth rates of the unstable and stable modes. Second, to what extent is the argument that small values of $D$ do not destabilize the system as a whole, an artifact of the stabilizing influence of the loworder numerics used by Siems et al. (1990) and Siems and Bretherton (1992)? To answer this question, a highorder numerical algorithm based on sixth-order compact schemes in space and a fourth-order five-step RungeKutta in time is proposed in section 4. Two-dimensional simulations are discussed in section 5 to extend the previous linear study into the nonlinear regime, showing the complicated pattern of mixing introduced by the buoyancy reversal - even for values $D \simeq 0.05$. At the same time, these simulations are used to validate the numerical algorithm and to further study the flow for the geophysically interesting case of $0<D \ll 1$; in particular, statistics related to the mixture fraction, the energy budget and entrainment are presented and discussed. It merits emphasis that the goal of the paper is not to derive results directly applicable to the real stratocumulustopped boundary layer (of the order of $10^{3} \mathrm{~m}$ ), but rather to investigate some elementary issues related to this question so as to gain insight to the phenomena occurring on a small scale (of the order of $10 \mathrm{~m}$ ) at the cloud top.

\section{Formulation}

The geometry considered is an unbounded two-layer system with the upper non-turbulent layer warmer and unsaturated and the lower one, also non-turbulent, cooler and supersaturated, gravity acting downwards. It is distinguished from (and simpler than) the stratocumulus-topped boundary layer (Lilly, 1968; Stevens, 2002) in which the finite-size bottom layer is turbulent. It is therefore an idealized problem and will be referred to as the cloudtop mixing layer, being shear-driven if Kelvin-Helmholtz instability dominates, buoyancy-driven if buoyancy reversal instability dominates, or a mixture of both. Only the shear-free configuration will be discussed in this paper.

Assuming that the liquid water phase can be represented as a continuum with the same diffusivity as the vapour, for low Mach number conditions the transport equations for the total-water specific humidity and the enthalpy reduce to the advection-diffusion equation. Given equal thermal and mass diffusivities and with appropriate boundary and initial conditions, the calculation of both can be represented in terms of the evolution of a single conserved scalar field, the mixture fraction $\chi(\mathbf{x}, t)$, satisfying the same advection-diffusion equation. In a two-layer system as we have here, the mixture fraction can be chosen to indicate the relative amount of mass of the fluid particle that originates from the upper layer. If thermodynamic equilibrium is assumed, the value of $\chi$ at each point and time determines completely the thermodynamic state of the fluid particle; in particular it provides the density as $\rho(\mathbf{x}, t)=\rho^{\mathrm{e}}(\chi(\mathbf{x}, t))$ for a function $\rho^{\mathrm{e}}(\chi)$ to be given.

In the Boussinesq limit with constant transport coefficients, the governing equations are then

$$
\begin{aligned}
\frac{\partial \mathbf{v}}{\partial t}+\nabla \cdot(\mathbf{v} \otimes \mathbf{v}) & =-\nabla p+v \nabla^{2} \mathbf{v}+b \mathbf{k}, \\
\nabla \cdot \mathbf{v} & =0, \\
\frac{\partial \chi}{\partial t}+\nabla \cdot(\mathbf{v} \chi) & =\kappa \nabla^{2} \chi,
\end{aligned}
$$

where the buoyancy is

$$
b=\frac{\rho_{0}-\rho}{\rho_{0}} g .
$$

In the equations above, $p$ is a modified pressure divided by the reference density, $v$ is the kinematic viscosity, $\kappa$ is the scalar diffusivity and $g$ represents the magnitude of the gravity acceleration, which is assumed constant and acting downwards along the vertical direction $\mathrm{Oz}$, i.e. $\mathbf{g}=-g \mathbf{k}$. Flow variables from the upper layer, where $\chi=1$, will be denoted by the subscript 1 , and subscript 0 will denote the lower layer, where $\chi=0$. The parameter

$$
b_{1}=\frac{\left(\rho_{0}-\rho_{1}\right)}{\rho_{0}} g
$$

quantifies the strength of the stable inversion. Positively buoyant means $b>0$ and the volumetric force is then directed upward along $\mathbf{k}$. 
The function $b^{\mathrm{e}}(\chi)$, or equivalently $\rho^{\mathrm{e}}(\chi)$, remains to be characterized to obtain the volumetric force from the relation $b(\mathbf{x}, t)=b^{\mathrm{e}}(\chi(\mathbf{x}, t))$. The first parameter entering is the density difference across the layer $\rho_{0}-\rho_{1}$, which is equivalent to a buoyancy value $b_{1}$ already introduced above. If the density were a linear function of the scalar, i.e. $\rho=\rho_{0}+\chi\left(\rho_{1}-\rho_{0}\right)$, then $b^{\mathrm{e}}(\chi)=b_{1} \chi$. When a phase change occurs and buoyancy reversal is present, linear thermodynamic analysis for a small density difference $\rho_{0}-\rho_{1}$ shows that this function can be well approximated by a piecewise-linear profile, characterized by a maximum density $\rho_{\mathrm{s}}$ at a particular mixture fraction value $\chi_{\mathrm{s}}$ that is fixed by the initial data. This maximum density is introduced in the problem through the non-dimensional buoyancy reversal parameter (Shy and Breidenthal, 1990)

$$
D=\frac{\rho_{\mathrm{s}}-\rho_{0}}{\rho_{0}-\rho_{1}}=-\frac{b_{\mathrm{s}}}{b_{1}} .
$$

Figure 1 shows the function $b^{\mathrm{e}}(\chi) / b_{1}$ for the cases described in Table I (to be discussed later in section 5), the exact equilibrium solution given by a polynomial fit to the vapour pressure function (Flatau et al., 1992). The approximate piecewise-linear behaviour is clearly revealed in that figure.

The discontinuity in the derivative observed in those curves at $\chi_{\mathrm{s}}$, which corresponds to just saturation conditions, needs to be smoothed if the high-order schemes normally employed in DNS and later described are to be used, since they are not monotone and the derivative of the momentum equation is needed for the pressure equation. Therefore, the buoyancy function is approximated as

$$
\begin{aligned}
\frac{b^{\mathrm{e}}(\chi)}{b_{1}}= & -\frac{D}{\chi_{\mathrm{s}}} \chi \\
& +\left(\frac{1+D}{1-\chi_{\mathrm{s}}}+\frac{D}{\chi_{\mathrm{s}}}\right) \delta_{\mathrm{s}} \ln \left[\exp \left(\frac{\chi-\chi_{\mathrm{s}}}{\delta_{\mathrm{s}}}\right)+1\right]
\end{aligned}
$$

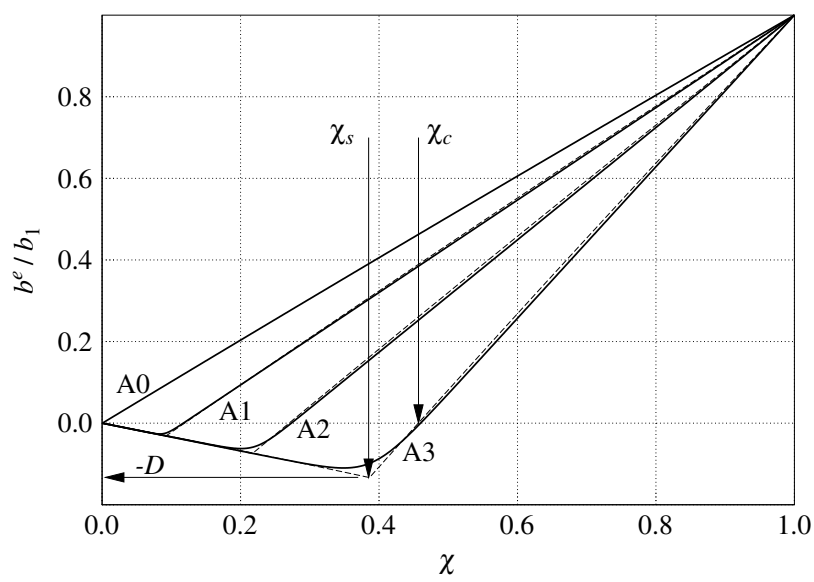

Figure 1. Non-dimensional buoyancy mixing function for data in Table I: exact thermodynamic equilibrium (dashed), and approximation from Equation (4) (solid). Parameters $D, \chi_{s}$ and $\chi_{c}$ are shown for case A3. which corresponds to the profile of the derivative $\mathrm{d} b^{\mathrm{e}} / \mathrm{d} \chi$ following a hyperbolic tangent between two different levels and centred at $\chi_{\mathrm{s}}$ (Figure 1). Mixture fractions smaller than $\chi_{\mathrm{c}}=\left(\chi_{\mathrm{s}}+D\right) /(1+D)$ are negatively buoyant. Numerical studies performed to calculate the influence of $\delta_{\mathrm{s}}$ on growth rates are presented in section 5.2 and show that a smoothing parameter defined by $\delta_{\mathrm{s}}=\chi_{\mathrm{s}} / 16$ leads to small enough deviations.

The equations are to be solved in a rectangular domain assuming periodicity in the horizontal directions $O x$ and $O y$. The boundary conditions imposed at the top and the bottom are zero normal velocity and zero normal derivative of the horizontal velocities and the scalar field $\chi$. The Neumann boundary conditions for the Poisson equation for the pressure at the top and the bottom are then (Gresho, 1991)

$$
\frac{\partial p}{\partial z}=v \frac{\partial^{2} w}{\partial z^{2}}+b
$$

where the boundary conditions on the velocity have been already applied and $w$ is the vertical velocity. Additionally, one reference value of $p$ (irrelevant for the flow) has to be given at one point.

If there is a velocity-scale $U_{0}$ externally imposed (e.g. from a mean shear or from a turbulent state in one of the layers) with a length-scale $L_{0}$, then dimensional analysis shows that the general solution can be written in the form

$$
\begin{aligned}
& \frac{\mathbf{v}(\mathbf{x}, t)}{U_{0}}=f\left(\frac{\mathbf{x}}{L_{0}}, \frac{t U_{0}}{L_{0}} ; \operatorname{Re}, \operatorname{Pr}, R i, \chi_{\mathrm{s}}, D, \frac{a}{L_{0}}, \frac{\delta}{L_{0}}\right), \\
& \chi(\mathbf{x}, t)=f^{*}\left(\frac{\mathbf{x}}{L_{0}}, \frac{t U_{0}}{L_{0}} ; \operatorname{Re}, \operatorname{Pr}, \operatorname{Ri}, \chi_{\mathrm{s}}, D, \frac{a}{L_{0}}, \frac{\delta}{L_{0}}\right),
\end{aligned}
$$

where the reference Reynolds and Richardson numbers are $R e=L_{0} U_{0} / \nu$ and $R i=b_{1} L_{0} / U_{0}^{2}$, respectively, and the Prandtl number is $P r=v / \kappa$. An amplitude $a$ of the initial perturbation of the interface has been assumed along with a initial thickness $\delta$ of the mean scalar profile of $\chi$.

If there is no velocity-scale externally imposed, then $b_{1}$ and $L_{0}$ can be used to write

$$
\begin{aligned}
& \frac{\mathbf{v}(\mathbf{x}, t)}{\sqrt{L_{0} b_{1}}}=f\left(\frac{\mathbf{x}}{L_{0}}, t \sqrt{\frac{b_{1}}{L_{0}}} ; G r, \operatorname{Pr}, \chi_{s}, D, \frac{a}{L_{0}}, \frac{\delta}{L_{0}}\right), \\
& \chi(\mathbf{x}, t)=f^{*}\left(\frac{\mathbf{x}}{L_{0}}, t \sqrt{\frac{b_{1}}{L_{0}}} ; G r, \operatorname{Pr}, \chi_{s}, D, \frac{a}{L_{0}}, \frac{\delta}{L_{0}}\right),
\end{aligned}
$$

as the general solution to the non-dimensional equations

$$
\begin{aligned}
\frac{\partial \mathbf{v}}{\partial t}+\nabla \cdot(\mathbf{v} \otimes \mathbf{v}) & =-\nabla p+\frac{1}{G r^{1 / 2}} \nabla^{2} \mathbf{v}+b \mathbf{k}, \\
\nabla \cdot \mathbf{v} & =0, \\
\frac{\partial \chi}{\partial t}+\nabla \cdot(\mathbf{v} \chi) & =\frac{1}{G r^{1 / 2} P r} \nabla^{2} \chi,
\end{aligned}
$$

which introduce the reference Grashof number (common in free convection flows, e.g. Tritton, 1988) as $G r=$ 
$L_{0}^{3} b_{1} / v^{2}$. Other combinations of these non-dimensional groups might be preferable for different particular configurations, as will be done in section 5 .

The unperturbed initial condition is assumed to correspond to the enthalpy and total specific humidity following an error function profile, which is a solution of the purely diffusive equations. This is imposed in the current formulation by prescribing the mixture fraction at the initial time as

$$
\chi_{i}(z)=\frac{1}{2}\left[1+\operatorname{erf}\left(\frac{z}{2 \delta}\right)\right] .
$$

The origin of the coordinate system is taken at the interface, and the thickness of this interface in terms of $\chi$ is parametrized by $\delta$. Evaporative cooling (Equation (4) and Figure 1) implies then a three-layer structure in the density field, with a middle heavier layer of an approximate thickness of order $\delta \chi_{c}$. The effect of a perturbation of magnitude $a$ to this configuration is further explored in the following sections.

\section{Linear stability analysis}

This section discusses the linear stability analysis of a three-layer density field without mean shear. The initial perturbation $a$ is assumed to be small enough, i.e. $a /\left(\delta \chi_{\mathrm{c}}\right) \ll 1$; stronger perturbations falling outside this linear regime will be considered in section 5 via numerical simulations. The basic configuration is depicted in Figure 2. It represents an idealized buoyancy reversal system, substituting the smooth density variation by a stepwise profile. The analysis is done assuming constant density in each of the three layers and irrotational flow. This problem, for the case of a two-layer system, is described in many textbooks (e.g. Turner, 1973) and the general multilayer case has been also discussed in the literature (Yang and Zhang, 1993). A three-layer system like the one here has received less attention; experimental investigations have been reported (Sutherland, 2002; Mehta et al., 2002; Jacobs and Dalziel, 2005), but all of them with a stratification different from the one considered here. It is therefore of interest to study this threelayer system in detail.

Let us denote with $\phi_{j}$ the velocity potential in each of the layers, which satisfies the Laplace equation, and

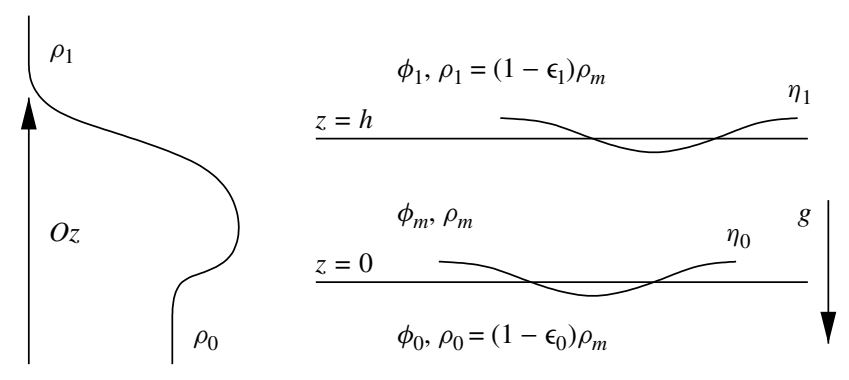

Figure 2. Vertical density profile (left) and three-layer idealized configuration (right) representing the buoyancy reversal state by a density $\rho_{m}$ greater than $\rho_{0}$ and $\rho_{1}$. with $\eta_{0}$ and $\eta_{1}$ the displacement of each interface with respect to the reference position, separated by a distance $h$ (Figure 2). The boundary conditions for the linearized problem consist of the kinematic condition,

$$
\begin{aligned}
& \frac{\partial \phi_{0}}{\partial z}=\frac{\partial \phi_{m}}{\partial z}=\frac{\partial \eta_{0}}{\partial t} \quad \text { at } \quad z=0, \\
& \frac{\partial \phi_{1}}{\partial z}=\frac{\partial \phi_{m}}{\partial z}=\frac{\partial \eta_{1}}{\partial t} \quad \text { at } \quad z=h,
\end{aligned}
$$

and the dynamic condition of continuity of pressure at the interfaces,

$$
\begin{aligned}
& \frac{\partial}{\partial t}\left(\rho_{m} \phi_{m}-\rho_{0} \phi_{0}\right)+\left(\rho_{m}-\rho_{0}\right) g \eta_{0}=0 \quad \text { at } \quad z=0, \\
& \frac{\partial}{\partial t}\left(\rho_{1} \phi_{1}-\rho_{m} \phi_{m}\right)+\left(\rho_{1}-\rho_{m}\right) g \eta_{1}=0 \quad \text { at } \quad z=h,
\end{aligned}
$$

having used the linearized Bernoulli equation in each of the three layers $\rho_{j} \partial \phi_{j} / \partial t+p_{j}+\rho_{j} g z=G_{j}$, with suitable functions $G_{j}(t)$.

Solutions are sought in the form

$$
\phi_{j}=f_{j}(z) \exp (\mathrm{i} k x+\sigma t), \quad j=0, m, 1 .
$$

The boundary condition on the normal velocity at the interfaces implies that $\eta_{0}=A_{0} \exp (\mathrm{i} k x+\sigma t)$ and similarly for $\eta_{1}$, along with relations between $f^{\prime}(z)$ at those interfaces. Applying these results to the solutions of the Laplace equations satisfied by $\phi_{j}$, we obtain

$$
\left(\begin{array}{c}
f_{0} \\
f_{m} \\
f_{1}
\end{array}\right)=\mathrm{e}^{-k z}\left(\begin{array}{c}
0 \\
\alpha_{m} \\
\alpha_{1}
\end{array}\right)+\mathrm{e}^{k z}\left(\begin{array}{c}
\beta_{0} \\
\beta_{m} \\
0
\end{array}\right)
$$

where the integration constants can be expressed in terms of $A_{0} \sigma / k$ and $A_{1} \sigma / k$ to write

$$
\left(\begin{array}{c}
f_{0} \\
f_{m} \\
f_{1}
\end{array}\right)=\frac{\sigma A_{1}}{k}\left(\begin{array}{c}
0 \\
\frac{\cosh (k z)}{\sinh (k h)} \\
-\mathrm{e}^{k(h-z)}
\end{array}\right)-\frac{\sigma A_{0}}{k}\left(\begin{array}{c}
-\mathrm{e}^{k z} \\
\frac{\cosh [k(h-z)]}{\sinh (k h)} \\
0
\end{array}\right) .
$$

Substituting these results in the boundary conditions for the pressure, we obtain a homogeneous linear system for the variables $A_{0}$ and $A_{1}$, with the characteristic secondorder polynomial for $\sigma^{2}$ :

$$
\begin{aligned}
\rho_{m}^{2}= & {\left[\sinh (k h)\left\{\frac{g k}{\sigma^{2}}\left(\rho_{m}-\rho_{0}\right)-\rho_{0}\right\}-\rho_{m} \cosh (k h)\right] } \\
& \times\left[\sinh (k h)\left\{\frac{g k}{\sigma^{2}}\left(\rho_{1}-\rho_{m}\right)-\rho_{1}\right\}-\rho_{m} \cosh (k h)\right] .
\end{aligned}
$$

The eigenvalue is defined as the square of the growth rate, $\sigma^{2}$. There are two eigenvalues and therefore two normal modes for each given wavenumber $k$ (Yang and Zhang, 1993).

Let us now introduce the ratios $\epsilon_{0}$ and $\epsilon_{1}$, such that

$$
\begin{aligned}
& \rho_{1}=\rho_{m}\left(1-\epsilon_{1}\right), \\
& \rho_{0}=\rho_{m}\left(1-\epsilon_{0}\right) .
\end{aligned}
$$


Then, the characteristic polynomial becomes

$$
\begin{aligned}
1= & \mathrm{e}^{2 k h}-\mathrm{e}^{k h} \sinh (k h)\left[\epsilon_{0}+\epsilon_{1}+\frac{g k}{\sigma^{2}}\left(\epsilon_{0}-\epsilon_{1}\right)\right] \\
& +\epsilon_{0} \epsilon_{1} \sinh ^{2}(k h)\left[1-\left(\frac{g k}{\sigma^{2}}\right)^{2}\right] .
\end{aligned}
$$

For the case $\epsilon_{0} \epsilon_{1} \neq 0$, the quadratic equation leads to

$$
\begin{aligned}
\frac{\sigma^{2}}{g k}= & \frac{\epsilon_{0} \epsilon_{1}}{\left(\epsilon_{1}-\epsilon_{0}\right)} \frac{1-\mathrm{e}^{-2 k h}}{1 \pm \sqrt{\Delta}}, \\
\Delta= & 1+4 \frac{\epsilon_{0} \epsilon_{1}}{\left(\epsilon_{1}-\epsilon_{0}\right)^{2}}\left(1-\mathrm{e}^{-2 k h}\right) \\
& \times\left[\left(1-\frac{\epsilon_{0}}{2}\right)\left(1-\frac{\epsilon_{1}}{2}\right)-\frac{\epsilon_{0} \epsilon_{1}}{4} \mathrm{e}^{-2 k h}\right] .
\end{aligned}
$$

In the Boussinesq limit, $O\left(\epsilon_{0}\right), O\left(\epsilon_{1}\right) \ll 1$ and the previous equation simplifies to

$$
\frac{\sigma^{2}}{g k}=\frac{\epsilon_{0} \epsilon_{1}}{\left(\epsilon_{1}-\epsilon_{0}\right)} \frac{1-\mathrm{e}^{-2 k h}}{1 \pm \sqrt{1+4 \frac{\epsilon_{0} \epsilon_{1}}{\left(\epsilon_{1}-\epsilon_{0}\right)^{2}}\left(1-\mathrm{e}^{-2 k h}\right)}} .
$$

In our particular case, $0<\epsilon_{0}<\epsilon_{1}$, and there is always one unstable solution $\sigma_{\mathrm{u}}$, corresponding to the positive sign in front of the square root, and one stable solution $\sigma_{\mathrm{s}}$ (two dispersive waves), corresponding to the negative sign. The physical system has therefore two time-scales, $\left|\sigma_{\mathrm{u}}\right|^{-1}$ and $\left|\sigma_{\mathrm{s}}\right|^{-1}$. Depending on the ratio $\left|\sigma_{\mathrm{u}}\right| /\left|\sigma_{\mathrm{s}}\right|$, the system will have fewer or more oscillations before the unstable mode is appreciated. The ratio between the unstable and stable eigenvalues is always less than 1, increasing with $\epsilon_{0} / \epsilon_{1}$ as shown in Figure 3. The dependence on the thickness of the unstable middle layer $h$ occurs through the non-dimensional wavenumber $k h$. For small values of $k h$, the unstable growth rate tends to zero, whereas the stable solution tends to $\sigma^{2}=$ $-\left(\epsilon_{1}-\epsilon_{0}\right) g k / 2 \simeq-k b_{1} / 2$, since $b_{1} \simeq g\left(\epsilon_{1}-\epsilon_{0}\right)$, this result corresponding to the oscillation of a two-layer system formed by layers 0 and 1 . For large values of

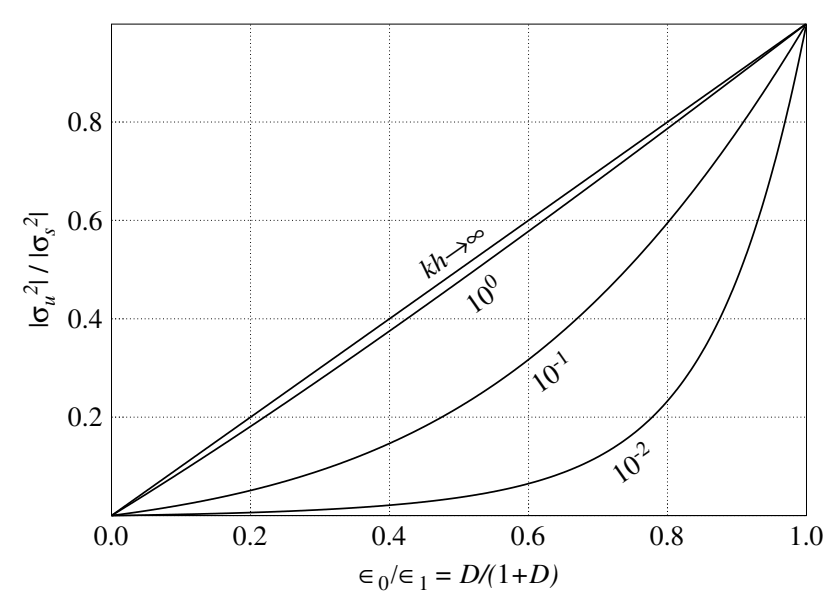

Figure 3. Ratio between the unstable and stable mode growth rates as a function of the density difference ratio and the non-dimensional wavenumber $k h$ in the Boussinesq limit $O\left(\epsilon_{0}\right), O\left(\epsilon_{1}\right) \ll 1$. $k h$, both growth rates in Equation (12) tend to those corresponding to two independent two-layer systems, one stable with eigenvalue $-\epsilon_{1} g k / 2$, the other one unstable with eigenvalue $\epsilon_{0} g k / 2$.

In case of $\epsilon_{0} \ll \epsilon_{1}$, as we expect to have, the solutions to Equation (12) are

$$
\begin{aligned}
& \sigma_{\mathrm{u}}^{2} \sim \epsilon_{0} g k\left(1-\mathrm{e}^{-2 k h}\right) / 2, \\
& \sigma_{\mathrm{s}}^{2} \sim-\epsilon_{1} g k / 2,
\end{aligned}
$$

which correspond to an oscillation period much smaller than the time-scale of the exponential growth of the unstable mode. The solution for $\sigma_{\mathrm{s}}$ corresponds to a stable two-layer configuration; the interface $\eta_{1}$ does not feel the small density difference at $z=0$. The solution for $\sigma_{\mathrm{u}}$ corresponds to an unstable two-layer system with a wall at a height $z=h$; this no-penetration condition is consistent with the fact that the upper interface $\eta_{1}$ oscillates fast compared with the characteristic time of the unstable layer, and only the mean position $z=h$ is felt by the interface $\eta_{0}$.

Equation (12) corresponds to the functional dependence anticipated by Equation (6) which we derived from dimensional analysis. This can be made explicit by noting that the former equation can be written in terms of the ratio $\epsilon_{0} / \epsilon_{1}$, the buoyancy reversal parameter (Equation (3)) is

$$
D=\frac{\epsilon_{0} / \epsilon_{1}}{1-\epsilon_{0} / \epsilon_{1}}
$$

when the density of the middle layer $\rho_{m}$ is identified with the saturation value $\rho_{s}$, and

$$
b_{1}=g \frac{\left(\epsilon_{1}-\epsilon_{0}\right)}{\left(1-\epsilon_{0}\right)} \simeq g\left(\epsilon_{1}-\epsilon_{0}\right)
$$

in the Boussinesq limit, leading to

$$
\frac{\sigma^{2}}{k b_{1}}=\frac{1}{4}\left[-1 \pm \sqrt{1+4 D(1+D)\left(1-\mathrm{e}^{-2 k h}\right)}\right]
$$

with $h$ playing the role of the thickness $\delta$ and $k$ the inverse of a characteristic length $L_{0}$. The stable solution corresponds again to the minus sign and the unstable to the plus sign. This result is valid for any value of $D$.

Equation (15) and Figure 3 allow one to compare the results obtained in the linear analysis with the discussion presented by Shy and Breidenthal (1990), Siems et al. (1990) and Siems and Bretherton (1992) about the role of the buoyancy reversal parameter $D$. In the first place, the instability condition $0<\epsilon_{0}<\epsilon_{1}$ translates with the new notation into $D>0$, which corresponds to the buoyancy reversal instability criteria introduced by those authors and equal to the nondimensionalized Randall-Deardorff criterion. Our analysis shows that this instability reflects one of two modes of the system, the second one being stable. Second, the critical value $D \simeq 1$ is reported by Siems and Bretherton (1992) to characterize the transition between buoyancy reversal instability and a 
strong buoyancy reversal instability, which can lead ultimately to cloud-top entrainment instability. However, the present linear analysis does not support such a scenario and Figure 3 depicts a rather smooth variation of the unstable growth rate with $D$. If the value $D=1$ is substituted in Equation (15), then the growth rate of the unstable mode is only a factor $\sqrt{2}$ smaller than the corresponding stable one for small wavelengths, hence $D \simeq 1$ should be simply interpreted as the condition for both modes to have comparable growth rates. This result suggests that to the extent $D \simeq 1$ represents a transition in the experiments of Shy and Breidenthal (1990), it does so because of nonlinear interactions related to the manner in which they perturb the interface in the experiment, or because of a finite-amplitude instability that cannot be described by the linear stability analysis. Finally, Equation (15) shows the influence of the thickness $h$ of the buoyancy reversal layer. For small values compared to the wavelength of the perturbation, the growth rates increase with $k h$, but asymptote to a constant after $k h$ is of order one.

Last, the case $D \ll 1$ (previous limit $\epsilon_{0} \ll \epsilon_{1}$ ) is of special interest because it occurs often in normal conditions at the top of the cloud deck (Kuo and Schubert, 1988; Stevens et al., 2003). In this limit

$$
\begin{aligned}
& \sigma_{\mathrm{u}}^{2} \sim k b_{1} D\left(1-\mathrm{e}^{-2 k h}\right) / 2, \\
& \sigma_{\mathrm{s}}^{2} \sim-k b_{1} / 2,
\end{aligned}
$$

and the ratio of growth rates depends on the square of the buoyancy reversal parameter

$$
\frac{\left|\sigma_{\mathrm{u}}\right|}{\left|\sigma_{\mathrm{s}}\right|} \sim \sqrt{D\left(1-\mathrm{e}^{-2 k h}\right)} .
$$

In brief, the linear stability analysis shows that the system is characterized by two modes, the stable one with a period of oscillation given by Equation (15) (negative sign), depending on the strength of the stable stratification as $k b_{1}$ and with corrections depending on $D$ and the non-dimensional wavenumber $k h$, and the unstable mode with a growth rate smaller than the stable one (Figure 3) and whose exact value is given by Equation (15) (positive sign). Two time-scales appear thus naturally from the equations, and a clear physical interpretation of the parameter $D$ for the case $D \ll 1$ typical of the stratocumulus top is obtained. The period of oscillation of the interfacial gravity wave can be written as $2 \sqrt{\pi} \sqrt{\lambda / b_{1}}$ if a wavelength $\lambda=2 \pi / k$ is introduced, and the characteristic time of the unstable mode scales as $D^{-1 / 2}$ times this oscillation period, according to Equation (17), with a prefactor depending on the nondimensional layer thickness $h / \lambda$. For $D=0.1$ the ratio of the growth rates is 0.3 and it increases monotonically with the thickness $h$, but asymptotically after $k h \simeq O(1)$.

\section{Numerical algorithm}

An incompressible code for solving the set of equations presented in section 2 has been derived from a previously existing finite-difference compressible code (Mellado et al., 2009). The algorithm follows Wilson et al. (1998); in particular, it uses a fourth-order low-storage RungeKutta scheme for the time advancement (Williamson, 1980) and sixth-order compact Padé schemes for the spatial derivatives (Lele, 1992) over a uniform grid, which provide the finite-difference approximations to the derivatives of a scalar field $p$, e.g. along the $O z$ direction, by solving the linear systems $\mathbf{A}_{1} \Delta_{z} p=\mathbf{B}_{1} p$ and $\mathbf{A}_{2} \Delta_{z z} p=\mathbf{B}_{2} p$, for the first- and second-order approximations, $\Delta_{z} p$ and $\Delta_{z z} p$, respectively. The matrices $\mathbf{A}_{i}$ are tridiagonal and the matrices $\mathbf{B}_{i}$ are pentadiagonal and the $0.1 \%$ error in the corresponding transfer function occurs at about 6 points per wavelength. The scheme is one-sided at the non-periodic boundaries (top and bottom).

The discrete Poisson equation for the pressure is written using Fourier decomposition inside the horizontal planes, which leads to

$$
\Delta_{z} \Delta_{z} \widehat{p}_{i j}-\lambda^{2} \widehat{p}_{i j}=\widehat{g}_{i j},
$$

where $\widehat{p}_{i j}$ is the vector formed by the horizontal Fourier modes

$i=0, \ldots,\left(\frac{N_{x}}{2}-1\right)$ and $j=\left(-\frac{N_{y}}{2}+1\right), \ldots,\left(\frac{N_{y}}{2}-1\right)$

(modes $N_{x} / 2$ and $N_{y} / 2$ are set to zero) at each z-position, and

$$
\lambda^{2}=\left[f\left(2 \pi i / N_{x}\right) /(\Delta x)\right]^{2}+\left[f\left(2 \pi j / N_{y}\right) /(\Delta y)\right]^{2},
$$

where $\Delta x$ and $\Delta y$ are the uniform grid spacing in the $O x$ and $O y$ directions, respectively. The transfer function $f(\omega)$ of the first-order finite-difference operator $\Delta_{z}$ needed in the equation above is (Lele, 1992)

$$
f(\omega)=\frac{(14 / 9) \sin (\omega)+(1 / 18) \sin (2 \omega)}{1+(2 / 3) \cos (\omega)} .
$$

The Neumann boundary conditions are obtained by Fourier transforming Equation (5). For the case $\lambda=0$, one of the Neumann boundary conditions has to be substituted by a Dirichlet one, and $\widehat{p}_{00}=0$ is used.

The difficulty resides in solving the sequence of discrete equations Equation (18), since each one is a linear system with a full matrix of size $N_{z} \times N_{z}$. The problem can be simplified by introducing an approximation to the operator $\Delta_{z} \Delta_{z}$ which leads to a system easier to solve. For instance, using the second-order finitedifference operator $\Delta_{z z}$, we have

$$
\Delta_{z z} \widehat{p}_{i j}-\lambda^{2} \widehat{p}_{i j}=\widehat{g}_{i j}-\mathbf{R} \hat{p},
$$

with

$$
\mathbf{R} p=\Delta_{z} \Delta_{z} p-\Delta_{z z} p=\left(\mathbf{A}_{1}^{-1} \mathbf{B}_{1} \mathbf{A}_{1}^{-1} \mathbf{B}_{1}-\mathbf{A}_{2}^{-1} \mathbf{B}_{2}\right),
$$

R being a full matrix. Cook and Dimotakis (2001) solve an approximation to this equation by neglecting the term 
$\mathbf{R} \hat{p}$ in their Rayleigh-Taylor turbulence simulations and solving the resulting pentadiagonal linear system, and the same approach is followed here. The error introduced by this step in solving the Poisson equation, due to the different truncation error between $\Delta_{z} \Delta_{z}$ and $\Delta_{z z}$, is easily analyzed in the case of periodic boundary conditions with help of the corresponding transfer functions. It is then observed that the error increases monotonically with the wavenumber and is of the order of $0.1 \%$ with 6 points per wavelength, which is consistent with the properties of the schemes used to calculate the derivatives of the equations. However, this step involves a non-zero dilatation error whose level depends on the resolution; the effects of this error will be discussed later in section 5 .

Finally, the buoyancy term in the momentum equation is prescribed in terms of the deviation $b^{\mathrm{e}}(\chi(\mathbf{x}, t))-$ $b^{\mathrm{e}}\left(\chi_{i}(z)\right)$, with $\chi_{i}$ from Equation (8). It is well known that any function $f(z)$ can be subtracted from $b(\mathbf{x}, t)$ with an appropriate redefinition of the scalar field $p$, and our particular choice reduces the gradient of $p$ at the upper boundary to almost zero, which contributes to well-behaved boundary conditions.

\section{Two-dimensional simulations}

Single-mode two-dimensional simulations are now presented with the purpose of illustrating the buoyancy reversal instability within the nonlinear regime as a complement to the linear stability analysis discussed before. These simulations also serve to validate the incompressible code for later use in the three-dimensional turbulence studies. The linear stability analysis identifies the possible significance of each of the parameters involved in the problem, namely, a time-scale related to $b_{1}$, a second time-scale which can be further related to $D$, and a thickness $h$ of the middle heavy layer. However, several assumptions underlying our linear analysis are not satisfied in reality: there is diffusion of the baroclinically produced vorticity, the shape of the initial density profile varies smoothly and there are finite amplitude effects. All of these effects can be captured in two-dimensional simulations, and hence these prove to be a useful next step in exploring whether the conclusions derived previously hold in reality.

No mean shear is considered and the initial perturbation is set by displacing sinusoidally the isosurface $\chi=0.5$ from the hydrostatic equilibrium over a wavelength $\lambda$ with an amplitude $a / 2$. The length-scale $a$ represents then the initial thickness of the mixing region. This initial condition is different from the disk anomaly used by Siems et al. (1990) and the single vortex employed by Siems and Bretherton (1992), but follows the common initialization employed in Rayleigh-Taylor configurations (Cook and Dimotakis, 2001; Mellado et al., 2005), the initial mechanical energy being introduced only through potential energy. The Prandtl number is unity.

\subsection{Mixture fraction}

A first series of simulations is performed to present here a qualitative description of the flow, and to study resolution requirements in subsection 5.2 for the numerical algorithm presented in section 4 . The geometrical parameters used are $\delta / \lambda=0.025$, where $\delta$ is the thickness of the initial error function profile (Equation (8)), and $(a / 2) / \lambda=0.1$, where $a / 2$ is the amplitude of the sinusoidal displacement. The reference box size in the vertical direction is $2 \lambda$; different sizes were also investigated (but not shown) to ensure that there are no finite-domain effects on the results presented here. The cases considered are described in Table I, where A1 is the reference case and corresponds to field experimental data of nocturnal marine stratocumulus from DYCOMS-II (Dynamics and Chemistry of Marine Stratocumulus; Stevens et al., 2003). The thermodynamic state of the upper layer is kept fixed at a temperature $T_{1}=19.1{ }^{\circ} \mathrm{C}$ and a total-water specific humidity $q_{t, 1}=1.5 \mathrm{~g} \mathrm{~kg}^{-1}$, and the lower state is modified to increase the effect of buoyancy reversal as shown in Figure 1. The case A0 does not retain evaporation, so that only the oscillating stable mode is present, and cases A2 and A3 consider an increasing buoyancy reversal imposed by means of a higher water content in the lower layer, keeping constant the density difference $\rho_{0}-\rho_{1}$ so that the period of the stable mode $2 \sqrt{\pi} \sqrt{\lambda / b_{1}}$ remains the same. The Boussinesq formulation only needs the values $\chi_{\mathrm{s}}$ and $D$ from this table.

Linear stability analysis has identified two time-scales in the problem. In this study we start from the stable case A0 and add increasingly the unstable mode, then it is reasonable to take $\sqrt{\lambda / b_{1}}$ as the reference time-scale for the non-dimensionalization. The relevant length-scale is the initial thickness of the mixing region, of order $a$, which leads to a viscous time-scale $a^{2} / \nu$. The reference Grashof number of the problem, defined in terms of the ratio between the two previous time-scales, is

$$
G r=\frac{a^{4} b_{1}}{v^{2} \lambda},
$$

Table I. Simulation series A.

\begin{tabular}{ccccccc}
\hline & $\begin{array}{c}q_{t, 0} \\
\left(\mathrm{~g} \mathrm{~kg}^{-1}\right)\end{array}$ & $\begin{array}{c}T_{0} \\
\left({ }^{\circ} \mathrm{C}\right)\end{array}$ & $\begin{array}{c}\epsilon_{1}-\epsilon_{0} \\
\left(\times 10^{-2}\right)\end{array}$ & $\begin{array}{c}\epsilon_{0} \\
\left(\times 10^{-3}\right)\end{array}$ & $D$ & $\chi_{\mathrm{s}}$ \\
\hline $\mathrm{A} 0$ & 8.0 & 10.5 & 2.54 & - & - & - \\
$\mathrm{A} 1$ & 9.0 & 10.6 & 2.54 & 0.79 & 0.031 & 0.09 \\
$\mathrm{~A} 2$ & 10.0 & 10.8 & 2.54 & 1.89 & 0.074 & 0.22 \\
$\mathrm{~A} 3$ & 12.0 & 11.3 & 2.54 & 3.37 & 0.133 & 0.39 \\
\hline
\end{tabular}

$q_{t, 0}$ and $T_{0}$ are the lower-layer total-water specific humidity and temperature.

$\epsilon_{0}$ and $\epsilon_{1}$ are defined by Equation (10) in terms of the densities, and $b_{1}=\left(\epsilon_{1}-\epsilon_{0}\right) /\left(1-\epsilon_{0}\right) g \simeq\left(\epsilon_{1}-\epsilon_{0}\right) g$. $D$ is the buoyancy reversal parameter from Equation (14), and $\chi_{\mathrm{s}}$ is defined in section 1 and Figure 1.

The upper-layer temperature and humidity are $19.1^{\circ} \mathrm{C}$ and $1.50 \mathrm{~g} \mathrm{~kg}^{-1}$ at pressure level $940 \mathrm{hPa}$. 

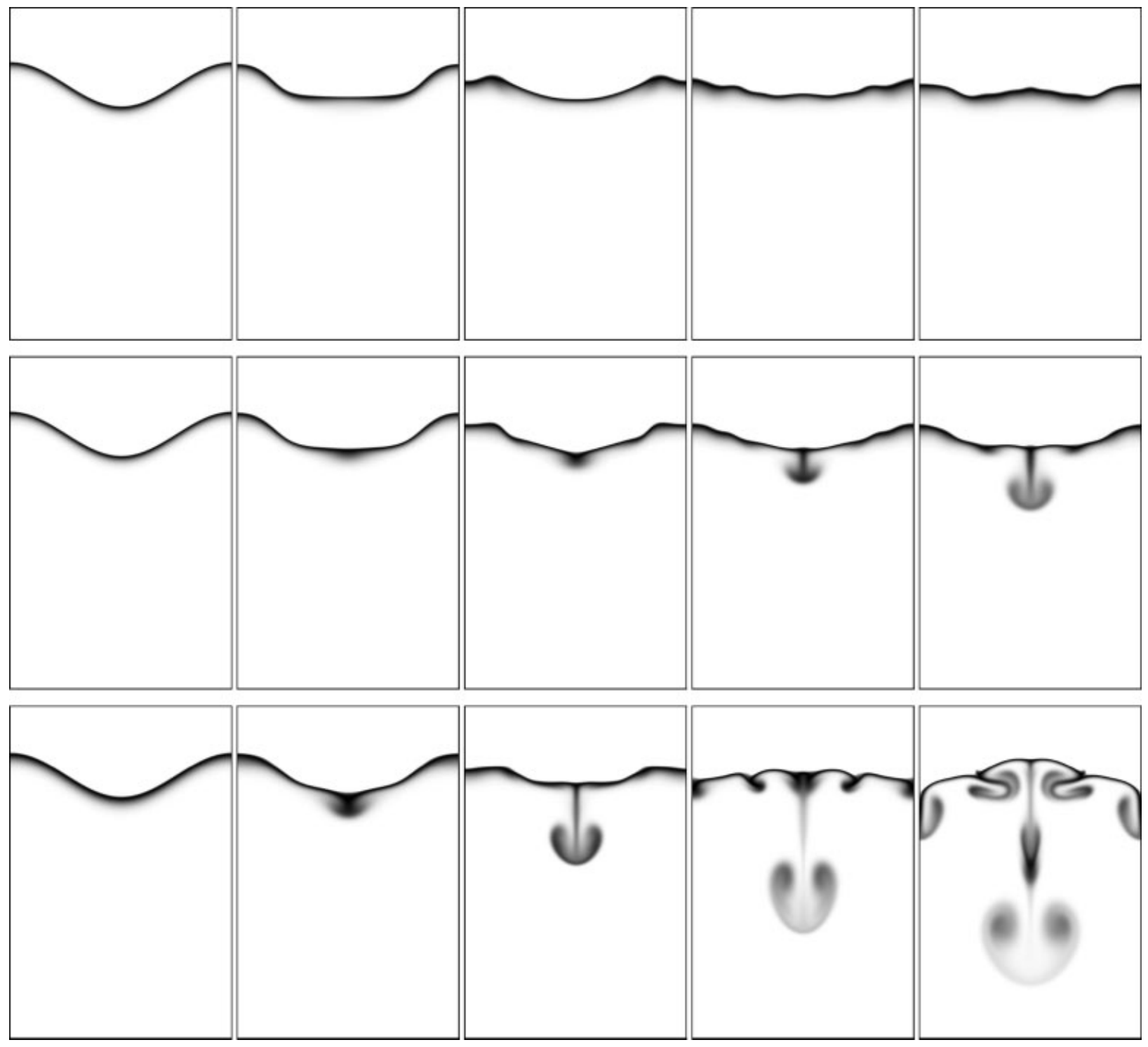

Figure 4. Negative buoyancy field for case A0 (top row), case A1 (middle row) and case A3 (bottom row) showing the evolution (left to right) starting from the initial condition and showing a frame every cycle of linear stable mode. Grashof number $G r=6.4 \times 10^{5}$.

and a value $G r=6.4 \times 10^{5}$ is used in this first series of simulations. The mesh of size $512 \times 1024$ is uniform and with equal grid spacing in every direction; resolution requirements are studied in detail in section 5.2.

Figure 4 shows qualitatively the evolution of the negative buoyancy field (points $\mathbf{x}$ for which $b(\mathbf{x}, t)<0$ are visualized using a grey scale, white for zero and black for minimum $\left.-D b_{1}\right)$ every $2 \sqrt{\pi} \sqrt{\lambda / b_{1}}$ time units. The stable case A0 is represented by $b^{\mathrm{e}}(\chi(\mathbf{x}, t))<0$ using the function $b^{\mathrm{e}}(\chi)$ corresponding to case $\mathrm{A} 1$ in order to compare the flow structure of both. The box height shown is only the lower $3 / 4$ of the domain employed in the simulation. The results obtained in the linear stability analysis are reproduced here: superimposed on a standing interfacial gravity wave, a falling finger, downdraught or spike, starts to form at the lowest point of the oscillation. This finger develops a mushroom shape typical of the Rayleigh-Taylor instability for small density differences (Sharp, 1984; Ramaprabhu and Andrews, 2004). On top of this process, viscosity tries to stop the motion while diffusion spreads the buoyancy reversal by mixing the ambient moist air with the less moist air inside the falling finger.

The non-homogeneous local mixing produced by the baroclinic production of vorticity at the oscillating inversion layer leads to the development of downdraughts at wavelengths smaller than the initial condition, which in turn enhances mixing at the core of the cloud-top mixing layer as compared to the non-evaporative case. This rearrangement of the buoyancy field is increasingly promoted as the buoyancy reversal and is augmented either by larger values of $D$ or $\chi_{\mathrm{s}}$, as noted by comparing cases A0, A1 and A3 in Figure 4. The time evolution presents a pulsating behaviour imposed by the stable mode, having the big initial central falling structure followed by a smaller one that appears in the last frame of Figure 4. A certain amount of heavy fluid is pumped periodically into the lower layer, the falling finger pinches off from 


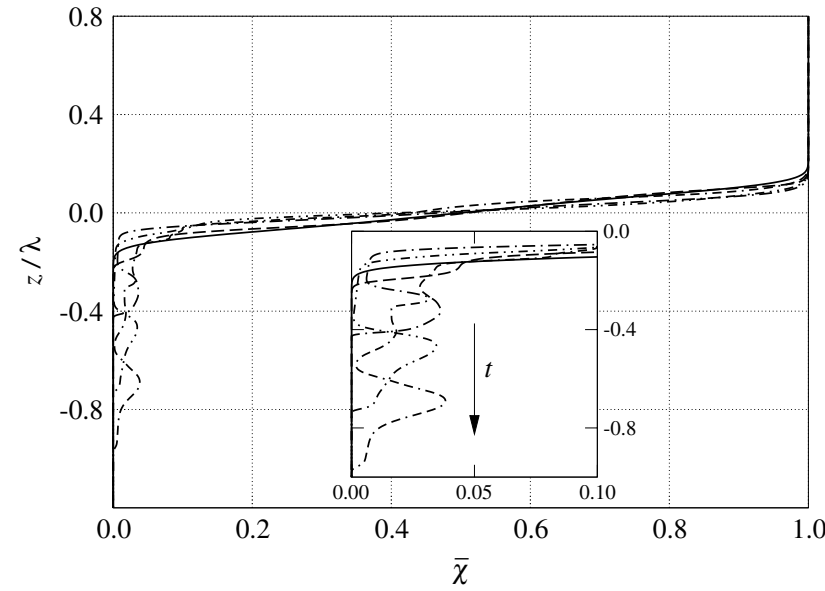

Figure 5. Vertical profile of the mean mixture fraction $\bar{\chi}$ for the case A3 every cycle of linear stable mode (the same times as shown in Figure 4): solid, dashed, dot-dashed, dot-dot-dashed and dot-dashdashed, respectively.

the oscillating central layer and the feed of fluid from this central layer ceases. It is observed that buoyancy reversal enhances mixing in the lower layer, but there is no strong instability in the sense of a runaway behaviour with a continuous tongue of falling upper fluid. This behaviour agrees with the laboratory experiments by Shy and Breidenthal (1990) and with the numerical simulations of Siems et al. (1990) and Siems and Bretherton (1992), who refer to it as a evaporative enhancement of entrainment and is characteristic of small buoyancy reversal parameters $D \ll 1$.

The instability process in now quantified with help of the mixture fraction $\chi$. Mean profiles $\bar{\chi}(z, t)$ for the case A3 are shown in Figure 5 and the perturbation growing into the lower layer depicted in Figure 4 is easily observed in these mean profiles. This perturbation can be located by the point where the field $\chi$ departs a given magnitude from the constant value of the corresponding layer. For example, for the falling finger, we can take $\bar{\chi}$ and scan from the lowest boundary upward until the mean profile reaches a given threshold; the distance from this point to the centre plane defines a height $h_{b}(t)$ of the falling finger. Normally, a threshold of $1 \%$ of the difference between the two layers is used in turbulent Rayleigh-Taylor cases, but mixing in this problem is mainly reduced to the small fraction of $\chi$ affected by buoyancy reversal; the inset in Figure 5 shows that $\bar{\chi}$ varies below 0.1 in the mixing region that forms in the lower layer. The threshold value 0.001 is therefore used.

The height $h_{b}$ of the falling finger as defined above is shown in Figure 6 as a function of time. The first thing to note is the superposition of the oscillating mode with the unstable mode. The effect of the buoyancy reversal parameters presented in Table I is also clearly exposed. As buoyancy reversal is increased from case A0 to A3, the middle unstable layer is relatively heavier (increasing $D$ ) and thicker (increasing $\chi_{\mathrm{s}}$ ), and therefore the growth rate of the unstable mode increases in comparison with the stable oscillation, the distance $h_{b}$ growing faster during the first 10-15 non-dimensional time units. These results

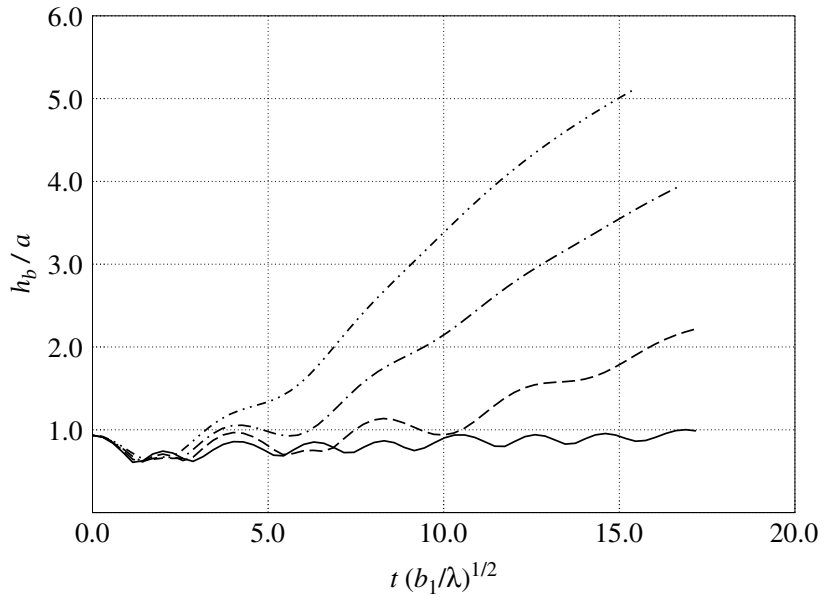

Figure 6. Temporal evolution of the penetration length $h_{b}$ of the downdraughts for the different cases of Table I: A0 (solid), A1 (dashed), A2 (dot-dashed), A3 (dot-dot-dashed).

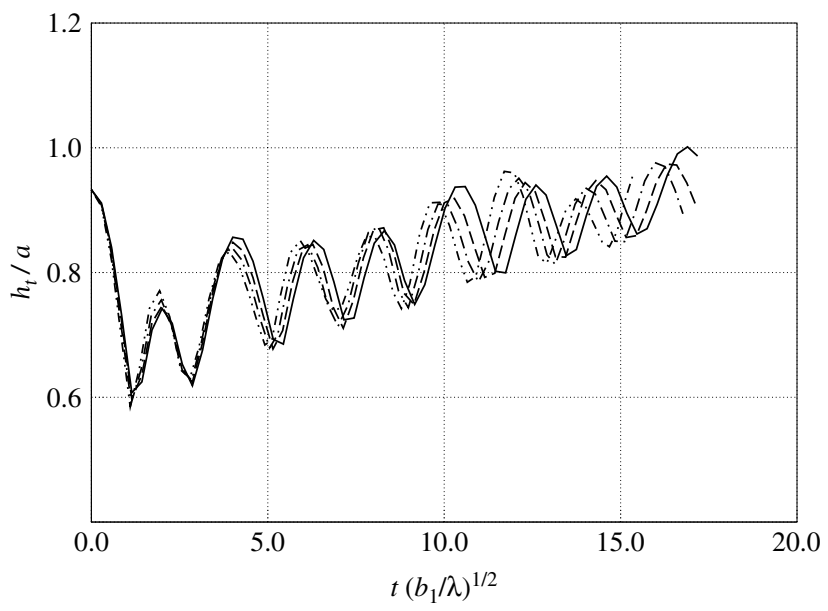

Figure 7. Temporal evolution of the upper perturbation thickness $h_{t}$ for the different cases of Table I: A0 (solid), A1 (dashed), A2 (dot-dashed), A3 (dot-dot-dashed).

agree with those obtained in the linear stability analysis of section 3 and presented in Figure 3. The stable case A0 only oscillates, with a period about $10 \%$ larger than that predicted by the linear theory and with a mean growth corresponding mainly to diffusion effects.

The same technique can be used to measure the thickness of the mixing region growing into the upper layer, $h_{t}(t)$, and the result is shown in Figure 7 . The strong difference with the evolution of the lower thickness $h_{b}(t)$ of Figure 6 is consistent with the mean profiles $\bar{\chi}$ shown in Figure 5 and the visualizations in Figure 4, $h_{t}$ showing only a steady mild growth of the mean level. This asymmetry in the vertical direction is a major difference with the conventional Rayleigh-Taylor configuration. It merits emphasizing that all cases present the same behaviour, and a strong buoyancy reversal as in case A3 does not differ qualitatively from the stable case A0. Since the mixture fraction is a conserved scalar,

$$
\frac{\mathrm{d}}{\mathrm{d} t} \int \bar{\chi} \mathrm{d} z=0
$$


holds for a long enough vertical interval. The fact that this series of simulations show no appreciable influence of increasing buoyancy reversal on $h_{t}$, but a strong one on $h_{b}$, implies that a stronger dilution of the downdraught occurs in the sequence $\mathrm{A} 0-\mathrm{A} 3$, as pointed out by one of the reviewers. Further discussion on this issue is presented in section 5.4.

An entrainment velocity with respect to the lower layer can be defined as the temporal derivative of $h_{b}(t)$. However, detailed quantitative results are influenced by the dominating stable periodic motion, the lack of multi-mode interaction and the two-dimensionality of the problem, thus we defer further analysis to threedimensional simulations, which are ongoing and will be discussed in detail in a forthcoming paper.

\subsection{Resolution requirements}

High resolution is needed to retain accurately the structure of the buoyancy field represented in Figure 1 within the turbulence interface formed between non-turbulent outer regions and the turbulent zone. In this respect, 6 points per vorticity thickness are normally used for a sixth-order compact scheme. The gradient thickness of a function $f$ varying monotonously across a difference $\Delta f$ between two levels is defined in terms of the maximum derivative by $\Delta f / f_{\max }^{\prime}$. For the case of the error function profile (Equation (8)) used here, this gradient thickness is $3.54 \delta$ and the resolution is then about 45 points per gradient thickness of the initial mixture fraction field $\chi$, which might seem too much. However, the peak of the density field occurs within a fraction $\chi_{\mathrm{s}}$ of the mixture fraction variation and the resolution of this buoyancy reversal layer can be as low as $10 \%$ of the complete viscous superlayer (Table I).

As the flow develops, the smallest scales in these twodimensional configurations correspond to the thickness of the diffusive layers, partially represented in Figure 4, which scale as $\left(G r^{1 / 2} P r\right)^{-1 / 2}$, but the particular resolution required in a simulation depends on the particular numerical algorithm. As already mentioned, there is a non-zero dilatation caused by the different truncation error between the first- and the second-order finite differences entering in the solution of the Poisson equation and this quantity can be used to monitor the accuracy of the simulation. Figure 8 shows the temporal evolution of the ratio between the $\mathrm{L}^{2}$-norms of the dilatation and the vorticity over the whole domain for the different cases.

The case without evaporative cooling $\mathrm{A} 0$ has a relative error $10^{-8}$ and it is therefore very well resolved (the minimum would be round-off error, $10^{-16}$, using an eightbyte representation of real numbers). The presence of buoyancy reversal introduces a dynamically active scale smaller than that observed in the mixture fraction field due to the mapping $b^{\mathrm{e}}(\chi)$, as already discussed, and the effect is observed clearly in Figure 8 because the dilatation error is increased in three orders of magnitude in comparison with the passive scalar case A0. With the resolution $512 \times 1024$ for the Grashof number $G r=$ $6.4 \times 10^{5}$ considered here, the dilatation error in the cases

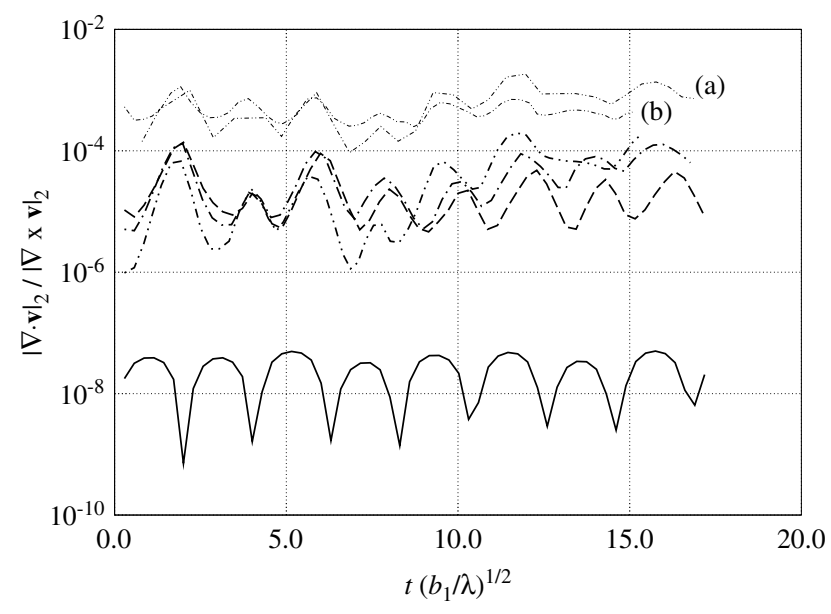

Figure 8. Temporal evolution of the dilatation error measured by the ratio between the $\mathrm{L}^{2}$-norms of the dilatation and vorticity. The thick lines are as in Figure 6 . The thin lines correspond to case A3 with: (a) half resolution, (b) no smoothing $\left(\delta_{\mathrm{s}}=0\right)$ in the buoyancy function $b^{\mathrm{e}}(\chi)$

with buoyancy reversal remains between $10^{-6}$ and $10^{-4}$ times the vorticity magnitude, which might be enough for small-scale analysis in the flow, like probability density functions of derivative fields. When half the resolution is employed, i.e. a mesh $256 \times 512$, then the dilatation error increases one order of magnitude; however, differences in the enstrophy fluctuation profile remain below $1 \%$ (penetration length $h_{b}(t)$ curves shown in Figure 6 are indistinguishable), which suggests that this mesh size is sufficient for the study of large-scale statistics like entrainment rate, Reynolds stress profiles or budgets of the corresponding transport equations.

As a conclusion from the previous results we can increase the reference Grashof number for the reference grid $512 \times 1024$ by a factor of 16 , according to the scaling based on $\left(G r^{1 / 2} P r\right)^{-1 / 2}$, and the dilatation error would be about $10^{-3}$ times the vorticity magnitude having still enough resolution to investigate large-scale quantities. Figure 9 corresponds to case A1 from Fig. 4 but with this new high Grashof number $G r=10^{7}$ plotted over a longer time. The large scale pattern is the same, but the development of stronger mixing just bellow the inversion layer is more clearly exposed, in addition to the richer small-scale details allowed by a larger Grashof number. High Grashof number simulations are further discussed below.

The effect of the smoothing parameter $\delta_{\mathrm{s}}$ employed in the definition of $b^{\mathrm{e}}(\chi)$ in Equation (4) has been also investigated and the dilatation curve for case A3 with the reference grid $512 \times 1024$ is included in Figure 8, with $\delta_{\mathrm{s}}=0$ instead the reference value $\delta_{\mathrm{s}}=\chi_{\mathrm{s}} / 16$. The effect is an increase in the dilatation error of one order of magnitude. On the other hand, the penetration length is higher by about $2 \%$ at the final time, which is a measure of the error introduced by the smoothing with $\delta_{\mathrm{s}}$ due to the small reduction in the minimum peak of the curve $b^{\mathrm{e}}(\chi)$ in Figure 1. 

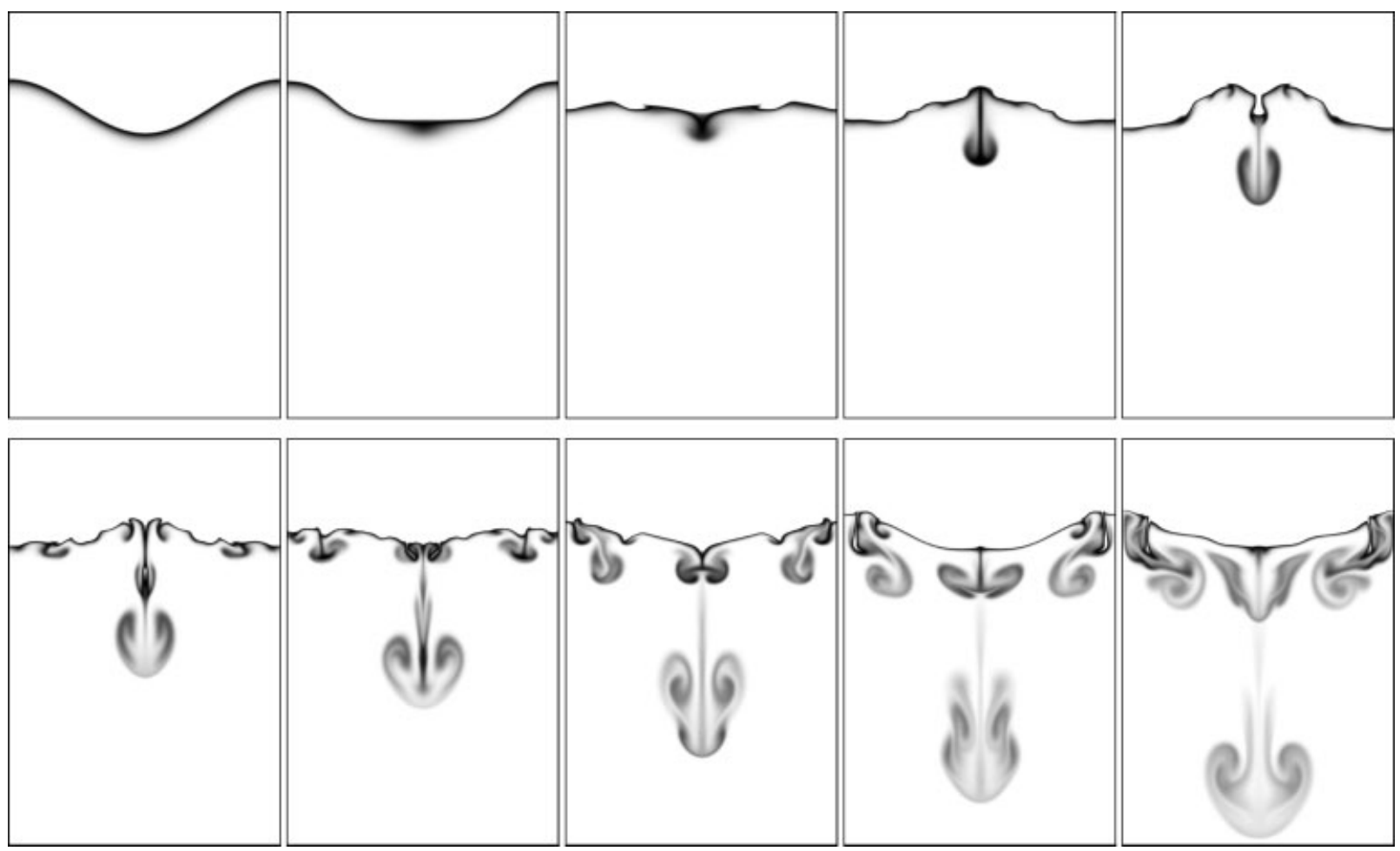

Figure 9. Evolution of the negative buoyancy field for case A1 as in Figure 4, but during a longer interval. The Grashof number $G r=10^{7}$.

\subsection{Energy budget}

The last statistics to be considered are those related to the energy budget. First, the potential energy is discussed in terms of the buoyancy function $b$, since the transport equations are normally written in terms of this variable. From the definition $b(\mathbf{x}, t)=b^{\mathrm{e}}(\chi(\mathbf{x}, t))$ and the advection-diffusion transport equation for $\chi$, we obtain the transport equation

$$
\frac{\partial b}{\partial t}+\nabla \cdot(\mathbf{v} b)=\kappa \nabla^{2} b+S,
$$

which shows that $b$ is not conserved but has a source term $S$. If this result is interpreted in terms of the density using Equation (2), it simply states that mass is not conserved, a well-known defect of the Boussinesq approximation (Lilly, 1996). The source term is

$$
S=-\frac{\mathrm{d}^{2} b^{\mathrm{e}}}{\mathrm{d} \chi^{2}} \kappa|\nabla \chi|^{2}=-\frac{\epsilon_{\chi}}{2} \frac{\mathrm{d}^{2} b^{\mathrm{e}}}{\mathrm{d} \chi^{2}}
$$

where $\epsilon_{\chi}=2 \kappa|\nabla \chi|^{2}$ is the so-called scalar dissipation rate and has dimensions of inverse time. When a piecewise-linear approximation is chosen to describe $b^{\mathrm{e}}(\chi)$, its second derivative is proportional to a delta function $\delta\left(\chi(\mathbf{x}, t)-\chi_{\mathrm{s}}\right)$. This case is formally equal to the Burke-Schumann solution of infinitely fast-reacting flows (Burke and Schumann, 1928; Peters, 2000), the buoyancy $b$ playing the role of one reactive scalar with the 'flame' sitting at the saturation surface $\chi(\mathbf{x}, t)=\chi_{\mathrm{s}}$. (This 'flame front' description has been already used qualitatively by Siems et al., 1990.) In our case $S$ is negative and we could talk about heat absorption instead of heat release. For the approximation expressed by Equation (4) employed here (already used in DNS of reacting flows by Pantano et al., 2003), the curvature of the buoyancy function is

$$
\frac{\mathrm{d}^{2} b^{\mathrm{e}}}{\mathrm{d} \chi^{2}}=b_{1}\left(\frac{1+D}{1-\chi_{\mathrm{s}}}+\frac{D}{\chi_{\mathrm{s}}}\right) \frac{1}{4 \delta_{\mathrm{s}}}\left[\cosh \left(\frac{\chi-\chi_{\mathrm{s}}}{2 \delta_{\mathrm{s}}}\right)\right]^{-2} .
$$

The term inside the first parentheses is the difference in the slopes of the piecewise-linear profile and represents the strength of the evaporative cooling, and the rest can be interpreted as a delta sequence that converges to the delta function as $\delta_{\mathrm{s}} \rightarrow 0$. A compromise in the value of $\delta_{\mathrm{s}}$, small enough to mimic a localized heat absorption zone but large enough to be resolved by the grid, has already been discussed in section 5.2.

Multiplying Equation (24) by the vertical coordinate $z$, we obtain

$$
\frac{\partial(b z)}{\partial t}+\nabla \cdot(\mathbf{v} b z)=b w+\kappa z \nabla^{2} b+z S
$$

and taking the mean over horizontal planes leads to

$$
\frac{\partial(\bar{b} z)}{\partial t}+\frac{\partial(\overline{b w} z)}{\partial z}=B+\kappa\left[\frac{\partial}{\partial z} z \frac{\partial \bar{b}}{\partial z}-\frac{\partial \bar{b}}{\partial z}\right]+z \bar{S}
$$

where the turbulent buoyancy flux is given by $B(z, t)=$ $\overline{w^{\prime} b^{\prime}}$ and the condition $\bar{w}=0$, satisfied in our configuration, has been used. The prime indicates turbulent fluctuations. The evolution equation for the total 
integrated potential energy is then

$$
\begin{aligned}
\frac{\mathrm{d}}{\mathrm{d} t}\left(-\int_{-\infty}^{\infty} z \bar{b} \mathrm{~d} z\right)= & -\int_{-\infty}^{\infty} B \mathrm{~d} z \\
& +\kappa b_{1}-\int_{-\infty}^{\infty} z \bar{S} \mathrm{~d} z
\end{aligned}
$$

The first line in the equation above corresponds to the balance we would obtain in the exact (mass-conserving) formulation of the problem: the integrated potential energy changes only through the total buoyancy flux. In contrast, in the Boussinesq limit we obtain two additional sources. Note that the balance in Equation (28) is independent of the reference used to measure vertical distances, but the time change of the integrated potential energy or the contribution from $S$ individually do depend on that arbitrary reference. Last, the integral of $\bar{b}$ as it is in the left-hand side does not converge since $\bar{b}=b_{1}$ far above in the upper layer. However, the equation can be written in terms of $\bar{b}-f(z)$ for any constant function $f(z)$ and we can choose $f(z)=b^{\mathrm{e}}\left(\chi_{i}(z)\right)$ in order to regularize the problem, $\chi_{i}$ from Equation (8), without loss of generality.

The turbulent buoyancy flux $B$ represents the transfer of energy between the turbulent kinetic energy and the potential energy, and it is easy to show that the total mechanical energy evolves according to the equation

$$
\begin{aligned}
\frac{\mathrm{d}}{\mathrm{d} t}\left[\int _ { - \infty } ^ { \infty } \left(\left|\mathbf{v}^{\prime}\right|^{2} / 2\right.\right. & -z \bar{b}) \mathrm{d} z]= \\
& -\int_{-\infty}^{\infty} \bar{\epsilon} \mathrm{d} z+\kappa b_{1}-\int_{-\infty}^{\infty} z \bar{S} \mathrm{~d} z
\end{aligned}
$$

where $\epsilon$ is the turbulent dissipation rate $\left(\epsilon=v v_{i, j}^{\prime}\left(v_{i, j}^{\prime}+\right.\right.$ $v_{j, i}^{\prime}$ ) in Cartesian coordinates using index notation). The term $\kappa b_{1}$ represents a linear diffusion source of potential energy which is always present, linear meaning that it contains the contribution from the linear part of the buoyancy function $b^{\mathrm{e}}(\chi)$ varying between 0 and $b_{1}$ (Equation (4) and Figure 1). Note that this term is always present, even in a one-dimensional purely diffusion case without buoyancy reversal, and it represents a constant linear growth in time. Further discussion can be found elsewhere, e.g. Winters et al. (1995), Peltier and Caulfield (2003). The source term $-\int z \bar{S} \mathrm{~d} z$ contains the nonlinear contribution, since $S$ is proportional to the curvature of the buoyancy function by its very definition in Equation (25).

A new series of simulations with a reference Grashof number $G r=10^{7}$ (series B described in Table II) has been performed in order to study the energy equation. The different cases are defined by varying each of the non-dimensional parameters identified through the paper instead of modifying the water content and temperature as was done in series A (Table I). The reference case is B1 and corresponds again to the experimental data taken from DYCOMS-II (Stevens et al., 2003), i.e. it is the same as case A1 with a higher Grashof number (Figure 9), whereas case B0 represents the stable configuration as A0 did. Cases B2 and B3 increase the buoyancy reversal by increasing $D$ and $\chi_{\mathrm{s}}$ separately, without modifying significantly the initial potential energy. On the other hand, cases B4 and B5 vary the initial conditions parameters $\delta / \lambda$ and $a / \lambda$, both decreasing the initial potential energy set in the system with respect to B1 and therefore diminishing the magnitude of the motion caused by the stable oscillations of the inversion. The vertical size of the domain has been extended to $2.5 \lambda$ in order to allow longer simulations, i.e. the grid size is $512 \times 1280$.

Figure 10 shows the balance of the integrated energy (Equation (29)) for case B1. It has been integrated in time to observe the accumulation of numerical errors,

$$
\begin{aligned}
& {\left[\int_{-\infty}^{\infty}\left(\frac{\left|\mathbf{v}^{\prime}\right|^{2}}{2}-z \bar{b}\right) \mathrm{d} z\right]_{0}^{t}=} \\
& \quad \kappa b_{1} t-\int_{0}^{t} \int_{-\infty}^{\infty} z \bar{S} \mathrm{~d} z \mathrm{~d} \tau-\int_{0}^{t} \int_{-\infty}^{\infty} \bar{\epsilon} \mathrm{d} z \mathrm{~d} \tau
\end{aligned}
$$

where the terms are, from left to right, the mechanical energy, the linear source of potential energy, the nonlinear source due to $S$, and the energy dissipation.

The results show that the linear source $\kappa b_{1}$ contributes positively to the overall balance, in particular increasing the potential energy through the diffusion of heavy fluid from the bottom to the top, and the rate of this contribution is constant in time and independent of the buoyancy reversal parameters $D$ and $\chi_{\mathrm{s}}$, and of the geometry of the initial condition $\delta$ and $a$. The other two terms contribute negatively: the nonlinear source term tends to reduce the potential energy because of the generation of negatively buoyant (heavy) parcels in the lower layer, and the turbulent dissipation because it represents the removal of turbulent kinetic energy by definition. The last curve in Figure 10 depicts the difference between the left-hand side and the righthand side of Equation (30), which quantifies the errors introduced by the numerical algorithm. It is observed that they are indeed negligible, decreasing to less than $1 \%$

Table II. Simulation series B.

\begin{tabular}{cccccc}
\hline & $D$ & $\chi_{s}$ & $\delta / \lambda$ & $(a / 2) / \lambda$ & $G r$ \\
\hline B0 & - & - & 0.025 & 0.10 & $10^{7}$ \\
B1 & 0.031 & 0.09 & 0.025 & 0.10 & $10^{7}$ \\
B2 & 0.062 & 0.09 & 0.025 & 0.10 & $10^{7}$ \\
B3 & 0.031 & 0.18 & 0.025 & 0.10 & $10^{7}$ \\
B4 & 0.031 & 0.09 & 0.050 & 0.10 & $10^{7}$ \\
B5 & 0.031 & 0.09 & 0.025 & 0.05 & $6.25 \times 10^{5}$ \\
\hline
\end{tabular}

Cases B2 and B3 increase buoyancy reversal with respect to the reference case $\mathrm{B} 1$.

Case B0 has no buoyancy reversal.

Cases B4 and B5 modify the initial conditions.

$\chi_{s}$ is defined in section 1 , and $a, \delta$ and $\lambda$ in section 5.1.

$G r$ is defined by Equation (22). 


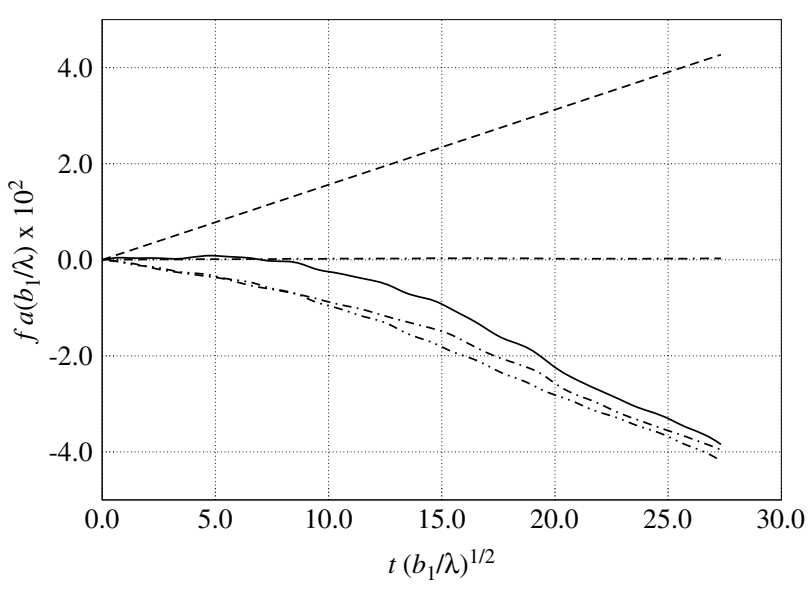

Figure 10. Energy budget according to Equation (30) for reference case B1: mechanical energy (solid), linear source (dashed), nonlinear source (dot-dashed), energy dissipation (dot-dot-dashed). Accumulated numerical error is indicated by the dot-dash-dashed line.

of the turbulent dissipation, and validates once more the numerical scheme used in the study.

The source term $S$ is further investigated. First, the accumulated integral value of the this source is computed, which from Equation (24) is related to the evolution of the integrated buoyancy

$$
\left[\int_{-\infty}^{\infty} \bar{b} \mathrm{~d} z\right]_{0}^{t}=-\int_{0}^{t} \int_{-\infty}^{\infty} \bar{S} \mathrm{~d} z \mathrm{~d} \tau .
$$

This term is shown in Figure 11 for the different cases of Table II, normalized by the strength of the evaporative cooling $b_{1}\left[(1+D) /\left(1-\chi_{\mathrm{s}}\right)+D / \chi_{\mathrm{s}}\right]$. With this normalization and from Equation (25), this term is approximately proportional to the accumulated integral value of the scalar dissipation rate $\epsilon_{\chi}$ conditioned on the evaporation surface $\chi(\mathbf{x}, t)=\chi_{\mathrm{s}}$, and therefore closely related to the turbulent mixing generated by the motion. In fact, the curves shown in Figure 11 show an increase in the accumulated buoyancy as more forcing is imposed, by increasing $D$ or $\chi_{\mathrm{s}}$ or $a$. The parameter $\delta$ is also consistent with this interpretation because the higher the $\delta$ for a given $a$, the smoother the initial buoyancy profile and the smaller the initial potential energy set in the problem.

Last, it is also interesting to calculate where in the domain the evaporative cooling is most concentrated, which is related to the source term of the energy equation. It has been observed in Figure 10 that the nonlinear source term $S$ decreases the integrated potential energy, but this interpretation really depends on the vertical reference position taken to calculate the potential energy, although the balance between them, which is equal to the remaining terms in Equation (28), is of course independent of that reference. A useful quantity for that purpose is the instantaneous centroid of the function $S(\mathbf{x}, t)$

$$
z_{\mathrm{s}}(t)=\frac{\int_{-\infty}^{\infty} z \bar{S} \mathrm{~d} z}{\int_{-\infty}^{\infty} \bar{S} \mathrm{~d} z}
$$

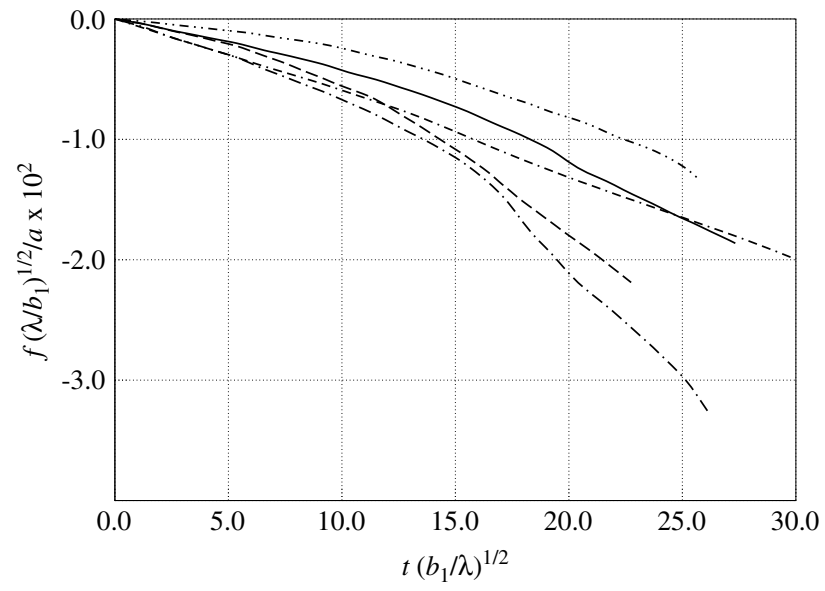

Figure 11. Normalized integrated buoyancy (Equation (31) and associated text) for different cases: reference, B1 (solid); double $D, \mathrm{~B} 2$ (dashed); double $\chi_{\mathrm{s}}, \mathrm{B} 3$ (dot-dashed); double $\delta, \mathrm{B} 4$ (dot-dot-dashed); half $a$, B5 (dot-dash-dashed)

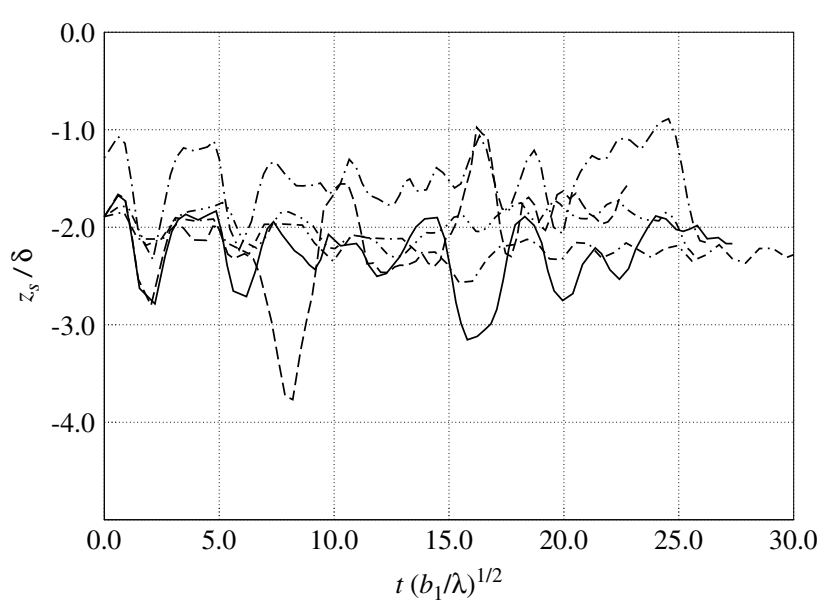

Figure 12. Centroid of the function $S(\mathbf{x}, t)$ according to definition Equation (32). The legend as in Figure 11.

This location can be used as reference for the definition of the potential energy, if desired, which allows Equation (28) to be written as

$$
\begin{aligned}
\frac{\mathrm{d}}{\mathrm{d} t}\left[-\int_{-\infty}^{\infty}\left\{z-z_{\mathrm{s}}(t)\right\} \bar{b} \mathrm{~d} z\right]= & \\
& \quad-\int_{-\infty}^{\infty} B \mathrm{~d} z+\kappa b_{1}+\frac{\mathrm{d} z_{\mathrm{s}}}{\mathrm{d} t} \int_{-\infty}^{\infty} \bar{b} \mathrm{~d} z,
\end{aligned}
$$

introducing a velocity $\mathrm{d} z_{\mathrm{s}} / \mathrm{d} t$. The energy Equation (29) can be correspondingly rewritten, if desired.

The evolution of $z_{\mathrm{S}}(t)$ is therefore of interest and it is shown in Figure 12 for the different cases. The first thing to note is that it is relatively constant in time, meaning that it presents oscillations but the mean remains constant. Hence, the mean position of the evaporative cooling source (and therefore the saturation surface $\left.\chi(\mathbf{x}, t)=\chi_{\mathrm{s}}\right)$ remains located between $1 \delta$ and $3 \delta$ below the reference position of the stable mode, and the decrease in the nonlinear source term $-\int z \bar{S} \mathrm{~d} z$ of the energy equation in Figure 10 is mainly determined by the 
evolution of the source term $S$, itself shown in Figure 11. After these parcels of relatively heavy mixture have been generated, mixing outside the saturation surface homogenizes the field $b$, mixing that can be generated either by the oscillating mode, dominant in the twodimensional single-mode cases considered in this paper, or by the downdraughts of the heavy mixtures themselves. The influence of $\delta$ in the problem has been determined, but the role of the other parameters cannot be really deduced from Figure 12 for the current configuration.

\subsection{Discussion}

The objective of this section has been the extension of the linear stability analysis into the nonlinear regime, explaining the flow characteristics based on the mixture fraction and energy statistics, and the validation of the incompressible code through a quantitative resolution study.

However, further discussion of the results seems appropriate at this point. One characteristic of turbulent entrainment is the mean entrainment rate, normally quantified either by a mean flow velocity towards the turbulent region, or by a displacement velocity of some mean profile towards the non-turbulent region (Hunt et al., 2006). In our problem, the mean vertical velocity is zero because of statistical homogeneity in the horizontal planes and the solenoidal character of the velocity field, and therefore we have discussed mean entrainment through the evolution of the penetration lengths $h_{b}$ and $h_{t}$ defined in terms of the mean vertical profile $\bar{\chi}(z, t)$ in section 5.1. Evaporative enhancement of turbulent entrainment is observed in Figures 6 and 4 and agrees with the general conclusions of Siems et al. (1990). However, the current results show that the motion below the cloud top for values of $D \ll 1$ is highly convoluted (cf. Figure 9) and suggest that in a three-dimensional broadband situation the flow could become turbulent only due to the buoyancy reversal instability without an additional sustained external forcing (e.g. from an already existing turbulent state in the lower layer or from local shear). It is not only weak circulations in the lower layer that need to be observed, and there is indeed a large difference between the stable cases and buoyancy reversing cases with small $D$, in contrast to previous suggestions (Siems et al., 1990; Siems and Bretherton, 1992). The difference is partly due to the low Reynolds numbers achieved in Siems et al. (1990), because they define a reference Reynolds number $\left(D b_{1} \lambda^{3} / v^{2}\right)^{1 / 2} \simeq 2 \times 10^{3}$, whereas the corresponding value here is about $10^{4}$. This increase in the affordable Reynolds number is due not only to the increase in grid size, but also to the employment of high-order schemes in our work. Siems and Bretherton (1992) report simulations with a reference Reynolds number $\left(b_{1} \lambda^{3} / v^{2}\right)^{1 / 2} \simeq 2 \times 10^{4}$, the equivalent value in our paper being $8 \times 10^{4}$. These two cases are more similar, yet they still report a negligible difference between the stable case and the case with $D=0.05$. The reason for their conclusion might be the low-order scheme they use, or the strong initial condition that they set with the initial vortex. We also note that the reference Grashof number $G r=a^{4} b_{1} /\left(v^{2} \lambda\right) \simeq 10^{7}$ used in this work is comparable to that found in the atmosphere when length-scales $\lambda$ between $1 \mathrm{~m}$ and $10 \mathrm{~m}$ are considered, as mentioned in the Introduction. For the reference case from DYCOMS-II, with a buoyancy $b_{1} \simeq 0.25 \mathrm{~m} \mathrm{~s}^{-2}$, the reference Grashof number varies approximately between $G r \simeq 10^{6}$ for $\lambda=$ $1 \mathrm{~m}$ and $G r \simeq 10^{9}$ for $\lambda \simeq 10 \mathrm{~m}$, using $a / \lambda=0.2$ and a kinematic viscosity of $1.5 \times 10^{-5} \mathrm{~m} \mathrm{~s}^{-2}$. The corresponding reference time-scales $\sqrt{\lambda / b_{1}}$ are $2 \mathrm{~s}$ and $6.3 \mathrm{~s}$, respectively. It is worth again stressing that the purpose of this work is the study of small-scale phenomena (of the order of 1-10m) at the cloud top, not the cloud-topped boundary layer as a whole, and therefore results cannot be directly extrapolated to the latter.

A second point to note is the difference between the effect of $D$ in the evolution of $h_{b}$ and $h_{t}$, Figures 6 and 7 , respectively. These figures show that there is no apparent enhancement of turbulent entrainment of upper-layer fluid, since all the curves in the latter figure develop approximately the same, and turbulent mixing enhancement by the evaporative cooling is restricted to the lower layer. This is consistent with the results of the study of the source term $S$ presented before. This source term concentrates around the saturation surface $\chi(\mathbf{x}, t)=\chi_{\mathrm{s}}$ and is such that the buoyancy field $b(\mathbf{x}, t)$ is always equal to $b_{\mathrm{s}}=-D b_{1}$ at that surface, and outside of it the field $b$ only mixes as a conserved scalar. Therefore, the motion of the system can be understood in terms of the moving surface $\chi(\mathbf{x}, t)=\chi_{\mathrm{s}}$ imposing the Dirichlet boundary condition $b(\mathbf{x}, t)=-D b_{1}$ and so creating negatively buoyant fluid and forcing the flow. (Recall that no other external forcing is considered in this work.) This surface moves randomly, but Figure 12 shows that its mean position remains at a constant height. This indicates that the turbulent motion brings fluid from the lower layer into contact with the saturation surface, where it acquires negative buoyancy locally by diffusion and forces further the turbulent motion in the lower layer, but there is no evidence of engulfment of pure fluid from the upper layer. This behaviour was anticipated by Siems et al. (1990), who referred to it as flame front mixing, as opposed to homogenization, where stirring by turbulence of engulfed above-cloud fluid dominates. Our simulations illustrate one case in which that first mechanism is prevalent and allows a turbulent flow to develop in the lower layer. We also observe that the saturation surface (or local instantaneous cloud top) remains at a constant height, whereas Siems et al. (1990) observed a downward motion of the mean position of isosurfaces of $\chi$, which in fact they use to define an entrainment velocity. We believe that difference is due to the excessive numerical dissipation in the algorithm they use, which generates more negatively buoyant fluid than it should.

In brief, the turbulent mixing promoted by the buoyancy reversal is mainly restricted to the lower layer, it is capped and constrained by the strong inversion and there is no enhancement of turbulent entrainment of the upper laminar layer into the mixing region; the mixing region mainly thickens downwards. The required thickening of 
the vertical profile $\bar{\chi}$ upwards due to the conservation of $\chi$ occurs mainly laminarly. How buoyancy reversal modifies an already existing turbulent entrainment caused, for instance, by an imposed shear or a lower turbulent layer remains an open question, but buoyancy reversal alone seems not to result in a runaway instability; it simply homogenizes the lower layer faster. The previous description suggests that the pure buoyancy-driven top-cloud mixing layer is similar to the upper boundary of a Rayleigh-Bénard convection configuration with this upper boundary free to move and the heat flux towards the lower layer dependent on that motion.

That said, it must be emphasized that the detailed quantitative analysis has necessarily to be discussed with three-dimensional broadband turbulent simulations because the mixing is different.

\section{Conclusions}

The buoyancy-driven cloud-top mixing layer has been investigated in this paper. Linear stability analysis of a simplified model consisting of three uniform inviscid layers with different densities has been presented. It identifies the two time-scales of the system, $\left|\sigma_{\mathrm{s}}\right|^{-1}$ for the stable mode and $\left|\sigma_{\mathrm{u}}\right|^{-1}$ for the unstable mode, shows the condition of buoyancy reversal instability $(D>0)$, and provides a new interpretation of the buoyancy reversal parameter $D$ as a measure of the ratio between those two time-scales. For small values of $D$, the growth rate of the unstable mode scales as $\sqrt{D}$ times that of the stable one. The effect of the second buoyancy reversal parameter $\chi_{\mathrm{s}}$ defining the mixture fraction of minimum buoyancy is retained through the dependence of the solution on the thickness of the intermediate layer, which can be estimated by $\delta\left(\chi_{\mathrm{s}}+D\right) /(1+D)$ if $\delta$ represents an appropriate thickness of the interface defined in terms of the mixture fraction field $\chi$. The unstable growth rate increases with respect to the stable growth rate monotonically with this thickness towards an asymptotic value.

The nonlinear regime has been illustrated through single-mode two-dimensional simulations, and these simulations qualitatively support the results from the linear stability analysis. This numerical study has also been used to explore quantitatively the consistency and convergence of the numerical methods employed. Spatial derivatives are based on sixth-order compact schemes, time advancement employs a forth-order low-storage RungeKutta algorithm and the Poisson equation is solved by Fourier transforming the equation in the horizontal periodic planes and then solving the resulting sequence of one-dimensional equations along the vertical direction, again using sixth-order compact schemes. The truncation error introduced by substituting the two first-order finite-difference operators by one second-order finitedifference, observed in the dilatation, has been used to discuss resolution requirements. The balance of the terms in the evolution equation of the integrated energy has also confirmed the accuracy of the numerical scheme, achieving a Grashof number $\mathrm{Gr}=10^{7}$, representative of a length-scale between 1 and $10 \mathrm{~m}$ in the real case, with a grid size $512 \times 1024$.

The two-dimensional simulations agree with previous work on the topic in that a runaway instability of the system does not become evident, but show that: (a) a turbulent flow in the lower layer can develop even for the cases with small values of $D$ without external forcing, and (b) a significant increase in the resolution of small-scale mixing is required, and hence the value of using high-order schemes. Statistics of the mixture fraction and energy budget show that there is no enhancement of turbulent entrainment of upper fluid by evaporative cooling for the low levels of buoyancy reversal typical of stratocumulus tops, and these heat absorption effects are mainly restricted to the lower layer. A formulation directly in terms of the buoyancy field which introduces a source term in the transport equation has been presented and the parallelism with infinitely fast-reacting flows discussed. It has also been shown that the associated buoyancy source term remains mainly localized below the inversion at a distance comparable to the initial thickness of the mixture fraction field, and the subsequent motion generated by the downdraughts dilutes these negatively buoyant parcels with the environmental fluid of the lower layer. Three-dimensional simulations are required to obtain more detailed quantitative results before considering the shear-driven case.

\section{Acknowledgement}

Partial financial support for this work was provided by the Deutsche Forschungsgemeinschaft within the SPP 1276 Metström program.

\section{References}

Burke SP, Schumann TEW. 1928. Diffusion flames. Ind. Eng. Chem. 20: $998-1004$.

Caughey SJ, Crease BA, Roach WT. 1982. A field study of nocturnal stratocumulus. II: Turbulence structure and entrainment. $Q$. J. R. Meteorol. Soc. 108: 125-144.

Cook AW, Dimotakis PE. 2001. Transition stages of Rayleigh-Taylor instability between miscible fluids. J. Fluid Mech. 443: 69-99.

Corrsin S, Kistler A. 1955. 'Free-stream boundaries of turbulent flows'. Technical Repor. 1244. NACA: Washington DC.

Deardorff JW. 1980. Cloud top entrainment instability. J. Atmos. Sci. 37: $131-147$.

Dimotakis PE. 2005. Turbulent mixing. Ann. Rev. Fluid Mech. 37: 329-356.

Fernando HJS. 1991. Turbulent mixing in stratified fluids. Ann. Rev. Fluid Mech. 23: 455-493.

Flatau PJ, Walko RL, Cotton WR. 1992. Polynomial fits to saturation vapor pressure. J. Appl. Meteorol. 31: 1507.

Grabowski WW. 1995. Entrainment and mixing in buoyancy-reversing convection with applications to cloud-top entrainment instability. Q. J. R. Meteorol. Soc. 121: 231-253.

Gresho PM. 1991. Incompressible fluid dynamics: some fundamental formulation issues. Ann. Rev. Fluid Mech. 23: 413-453.

Hunt JCR, Eames I, Westerweel J. 2006. Mechanics of inhomogeneous turbulence and interfacial layers. J. Fluid Mech. 554: 499-519.

Jacobs JW, Dalziel SB. 2005. Rayleigh-Taylor instability in complex stratifications. J. Fluid Mech. 542: 251-279.

Kuo H, Schubert WH. 1988. Stability of cloud-topped boundary layers. Q. J. R. Meteorol. Soc. 114: 887-916. 
Lele SK. 1992. Compact finite difference schemes with spectral-like resolution. J. Comput. Phys. 103: 16-42.

Lilly DK. 1968. Models of cloud-topped mixed layers under strong inversion. Q. J. R. Meteorol. Soc. 94: 292-309.

Lilly DK. 1996. A comparison of incompressible, anelastic and Boussinesq dynamics. Atmos. Res. 40: 143-151.

Mehta AP, Sutherland BR, Kyba PJ. 2002. Interfacial gravity currents. II: Wave excitation. Phys. Fluids 14: 3558-3569.

Mellado JP, Sarkar S, Zhou Y. 2005. Large-eddy simulation of Rayleigh-Taylor turbulence with compressible miscible fluids. Phys. Fluids 17: 76-101.

Mellado JP, Wang L, Peters N. 2009. Gradient trajectory analysis of a scalar field with external intermittency. J. Fluid Mech. in press.

Pantano C, Sarkar S, Williams FA. 2003. Mixing of a conserved scalar in a turbulent reacting shear layer. J. Fluid Mech. 481: 291-328.

Peltier WR, Caulfield CP. 2003. Mixing efficiency in stratified shear flows. Ann. Rev. Fluid Mech. 35: 135-167.

Peters N. 2000. Turbulent combustion. Cambridge University Press: Cambridge, UK.

Ramaprabhu P, Andrews MJ. 2004. Experimental investigation of Rayleigh-Taylor mixing at small Atwood numbers. J. Fluid Mech. 502: 233-271.

Randall DA. 1980. Conditional instability of the first kind upside-down. J. Atmos. Sci. 37: 125-130.

Sharp DH. 1984. An overview of Rayleigh-Taylor instability. Physica D 12: 3-18.

Shy SS, Breidenthal RE. 1990. Laboratory experiments on the cloudtop entrainment instability. J. Fluid Mech. 214: 1-15.

Siems ST, Bretherton CS. 1992. A numerical investigation of cloud-top entrainment instability and related experiments. Q. J. R. Meteorol. Soc. 118: $787-818$.
Siems ST, Bretherton CS, Baker MB, Shy S, Breidenthal RE. 1990. Buoyancy reversal and cloud-top entrainment instability. Q. J. R. Meteorol. Soc. 116: 705-739.

Stevens B. 2002. Entrainment in stratocumulus-topped mixed layers. Q. J. R. Meteorol. Soc. 128: 2663-2690.

Stevens B, Lenschow DH, Faloona I, Moeng CH, Lilly DK, Blomquist

B, Vali G, Bandy A, Campos T, Gerber H, Haimov S, Morley B, Thorton C. 2003. On entrainment rates in nocturnal marine stratocumulus. O. J. R. Meteorol. Soc. 129: 3469-3493.

Sutherland BR. 2002. Interfacial gravity currents. I: Mixing and entrainment. Phys. Fluids 14: 2244-2254.

Tritton DJ. 1988. Physical fluid dynamics. Oxford Science Publications. Turner JS. 1973. Buoyancy effects in fluids. Cambridge University Press.

Williamson JH. 1980. Low-storage Runge-Kutta schemes. J. Comput. Phys. 35: 48-56.

Wilson RV, Demuren AO, Carpenter M. 1998. 'Higher-order compact schemes for numerical simulation of incompressible flows'. Technical Report CR-1998-206922, NASA Langley Research Center: Springfield, Virginia, USA.

Winters KB, Lombard PN, Riley JJ, D’Asaro EA. 1995. Available potential energy and mixing in density-stratified fluids. J. Fluid Mech. 289: 115-128.

Wunsch S. 2003. Stochastic simulations of buoyancy reversal experiments. Phys. Fluids 15: 1442-1456.

Yamaguchi T, Randall DA. 2008. Large-eddy simulation of evaporatively driven entrainment in cloud-topped mixed layers. J. Atmos. Sci. 65: 1481-1504.

Yang Y, Zhang Q. 1993. General properties of a multilayer stratified fluids systems. Phys. Fluids A 5: 1167-1181. 